Title

\title{
Supramolecular assembly of the E. coli Ldcl upon acid stress
}

\author{
Authors \\ Matthew Jessop*, Clarissa Liesche*, Jan Felix*, Ambroise Desfosses, Megghane Baulard, Virgile \\ Adam, Angélique Fraudeau, Karine Huard, Grégory Effantin, Jean-Philippe Kleman, Maria Bacia- \\ Verloop, Dominique Bourgeois, Irina Gutsche ${ }^{\#}$ \\ ${ }^{1}$ Institut de Biologie Structurale, Univ Grenoble Alpes, CEA, CNRS, IBS, 71 Avenue des martyrs, F- \\ 38044 Grenoble, France \\ *: equal contribution, \#: corresponding author.
}

\section{Significance statement}

Bacteria possess a sophisticated arsenal of defence mechanisms that allow them to survive in adverse conditions. Adaptation to acid stress and hypoxia is crucial for the enterobacterial transmission in the gastrointestinal tract of their human host. When subjected to low $\mathrm{pH}$, E. coli and many other enterobacteria activate a proton-consuming resistance system based on the acidstress inducible lysine decarboxylase Ldcl. Here we develop generally-applicable tools to uncover the spatial localisation of Ldcl inside the cell by super-resolution fluorescence microscopy, and investigate the in vitro supramolecular organisation of this enzyme by cryo-EM. We build on these results to propose a mechanistic model for Ldcl function and offer tools for further in vivo investigations.

\section{Abstract}

Pathogenic and commensal bacteria often have to resist the harsh acidity of the host stomach. The inducible lysine decarboxylase Ldcl buffers the cytosol and the local extracellular environment to ensure enterobacterial survival at low $\mathrm{pH}$. Here, we investigate the acid-stress response regulation of $E$. coli Ldcl by combining biochemical and biophysical characterisation with negative stain and cryo-electron microscopy, and wide-field and super-resolution fluorescence imaging. Due to deleterious effects of fluorescent protein fusions on native Ldcl decamers, we opt for three-dimensional localisation of nanobody-labelled endogenous wild-type Ldcl in acid-stressed E. coli cells, and show that it organises into distinct patches at the cell periphery. Consistent with recent hypotheses that in vivo clustering of metabolic enzymes often reflects their polymerisation as a means of stimulus-induced regulation, we show that Ldcl assembles into filaments in vitro at physiologically relevant low $\mathrm{pH}$. We solve the structures of these filaments and of the Ldcl decamer formed at neutral $\mathrm{pH}$ by cryo-electron microscopy, and reveal the molecular determinants of Ldcl polymerisation, confirmed by mutational analysis. Finally, we propose a model for Ldcl function inside the enterobacterial cell, providing a structural and mechanistic basis for further investigation of the role of its supramolecular organisation in the acid stress response. 


\section{Introduction}

Cell survival requires the adaptation of metabolism to changing environmental demands. Biochemical regulation of metabolic enzymes by cellular metabolites has been intensely studied for many decades. In addition, a growing body of recent light microscopy observations highlights the spatial regulation of enzymes by stimulus-induced phase separation into distinct loci - liquid droplets, amyloid-like aggregates or ordered polymers (1). In eukaryotes, the observed condensation of fluorescently-labelled metabolic enzymes is triggered by stress, including medium acidification, hypoxia and nutrient limitation. Enterobacteria such as Escherichia coli, Salmonella and Vibrio encounter these types of conditions in the host gastrointestinal tract (2). One of the key enterobacterial proteins expressed during the acid stress response, upon oxygen limitation and regulated by the nutrient stress alarmone guanosine tetraphosphate (ppGpp), is the acid stressinducible lysine decarboxylase Ldcl (3-6). Ldcl has been scrutinised since the early 1940s because of direct links between the efficiency of the acid stress response and pathogenicity (7-9). This enzyme transforms lysine into cadaverine while consuming protons and producing $\mathrm{CO}_{2}$, thereby contributing to buffering of the bacterial cytosol and the extracellular medium under acid stress conditions to promote bacterial survival. While both the structure and the function of Ldcl have been thoroughly studied $(6,10)$, nothing is known about its localisation inside the bacterial cell, because its fluorescent protein fusions were previously described to form inclusion bodies (11).

Whereas the overwhelming majority of super-resolution fluorescence imaging is focused on eukaryotes, optical studies of bacterial systems are nearly exclusively centred on large macromolecular complexes with obvious superstructure such as cytoskeletal, cell division, chromosome partitioning, RNA degradation and secretion machineries (12-14). However, the relevance of the documented patchy or long-range helical localisation of some of these assemblies is now questioned and requires re-evaluation. Indeed, the vast majority of these studies were based on labelling with either fluorescent proteins or epitope tags shown to be able to induce artefactual associations and localisations (13-16).

A handful of examples of regulation of bacterial metabolic enzymes by phase separation through stimulus-triggered polymerisation concern well-conserved oligomeric proteins involved in nucleotide and amino acid metabolism, such as CTP synthase $(17,18)$ and glutamine synthetase (19). Interestingly, these enzymes are also able to assemble into filaments in vitro, and their in vivo condensates, detected both in bacteria and in eukaryotes, have been suggested to correspond to the polymerised state of the enzymes. Other examples of bacterial metabolic enzymes purified as polymers from bacterial extracts or forming polymers in vitro are the aldehyde-alcohol dehydrogenase AdhE (20) and the hydrogen-dependent $\mathrm{CO}_{2}$ reductase HDCR (21).

Specific to bacteria, $\mathrm{Ldcl}$ is a decamer composed of five dimers tightly arranged into pentameric double-rings (PDB ID: 3N75) (6). Interestingly, in vitro these double-rings were observed by negative stain electron microscopy (ns-EM) to stack on top of one another into filament-like structures $(6,8)$ but only at non-physiological pH below 5 , under which Ldcl should not be significantly expressed. Here, to address the spatial localisation of the $E$. coli Ldcl, we start by critically evaluating labelling artefacts with an ambition to define the optimal constructs for subsequent chromosomal manipulations. To this end, we overexpress and purify different 
fluorescent protein (FP) fusions of Ldcl, perform their structural and biochemical characterisation in vitro, and observe the distribution of the overexpressed constructs in vivo. This methodological section enables us to propose a workflow that brings together examinations of in vivo protein localisations with in vitro biochemical and ns-EM characterisations of the purified FP fusions in order to ensure artefact-free optical imaging investigations. This analysis is followed by unveiling a patchy distribution of endogenous $\mathrm{Ldcl}$ inside the $E$. coli cell upon acid stress. Because this condensation of Ldcl into patches is reminiscent of stress-induced polymerisation, in particular in yeast, we investigate the in vitro architecture of $\mathrm{Ldcl}$ at $\mathrm{pH} 5.7$. We show that at this physiological $\mathrm{pH}$, corresponding to the optimum of the Ldcl enzymatic activity, Ldcl is a polymer assembled of stacked decamers, and solve the polymer structure by cryo-electron microscopy (cryo-EM). For comparison, we also determined the cryo-EM structure of the Ldcl decamer at neutral $\mathrm{pH}$. In addition, mutational analysis of the Ldcl stack-forming interfaces allowed identification of critical residues involved in stack formation. Finally, we discuss the observed Ldcl localisation pattern in the light of the wealth of available functional and imaging data, and offer a structural and mechanistic basis of supramolecular Ldcl assembly, which will aid in the design of future experiments linking Ldcl stack formation to E. coli acid stress fitness.

\section{Results}

Fluorescent protein fusions affect Ldcl structure without modifying localisation of the overexpressed fusion constructs

Because of the small size of the E. coli cell, we opted for super-resolution microscopy imaging (22) and set out to localise Ldcl inside the cell upon acid stress by either Photoactivation Localisation Microscopy (PALM) or Stochastic Optical Reconstruction Microscopy (STORM) (2325). A priori, PALM seemed more relevant because this technique relies on genetically-encoded FPs fused to the protein of interest, and is therefore unmatched in terms of labelling specificity and efficiency. In addition, PALM does not require delivery of fluorescent molecules across the cell wall, and enables live cell imaging and single molecule tracking.

Considering that $\mathrm{Ldcl}$ is an acid stress response enzyme, we opted for either mGeosM (26) or Dendra2 ${ }_{\text {T69A (27) }}$ as FP markers because of their relatively low pKa values, high monomericity and high fluorescent quantum yields. In the $\mathrm{Ldcl}$ decamer structure, the $\mathrm{N}$-termini are oriented inwards and towards the central pore of the double ring, while the C-termini point to the ring periphery and are readily accessible from the outside. Therefore, on the one side, one could assume that an $\mathrm{N}$ terminal labelling with an FP would be likely to interrupt the Ldcl tertiary structure. On the other side, the only well characterised binding partner of Ldcl, the AAA+ ATPase RavA, is known to interact precisely with the C-terminal $\beta$-sheet of $\mathrm{Ldcl}$ (28). Assembly of two copies of doublepentameric rings of $\mathrm{Ldcl}$ and five copies of hexameric RavA spirals results in a huge 3.3 MDa macromolecular cage of intricate architecture and largely unknown function (28-30). The LdclRavA cage is proposed to assist assembly of specific respiratory complexes in $E$. coli and to counteract acid stress under starvation conditions by alleviating ppGpp-dependent inhibition of Ldcl (31-33). Thus, although these functions still require further investigation, preservation of the RavA-binding propensity should be one of the criteria for assessing the suitability of an Ldcl-FP fusion. Therefore, both $\mathrm{N}$ - and C-terminal fusion constructs with either mGeosM or Dendra2 ${ }_{\text {T69A }}$ 
attached to Ldcl via an appropriate linker were cloned into dedicated plasmids and overexpressed at conditions optimised for Ldcl overproduction (see Methods for details). Expression of fusion proteins was immediately detected by wide-field fluorescence imaging that showed a similar distribution for the four fusions (Figure 1A). Each construct was then purified in order to assess its structural integrity and RavA binding capacity in vitro, with a goal of defining the most suitable construct for the subsequent creation of a corresponding chromosomal fusion.

The N-terminal Dendra2 ${ }_{T 69 A}$-Ldcl fusion formed exclusively dimers, confirming the structurebased prediction that the fluorescent tag at this position would perturb the dimer-dimer interaction (Figure 1B, Supplementary Figure 1). Admittedly, native Ldcl was shown to dissociate into dimers in vitro at $\mathrm{pH}$ above approximately 7.5 , but in this $\mathrm{pH}$ range $\mathrm{Ldcl}$ is not supposed to be expressed in the cell and therefore this dissociation may be irrelevant. Surprisingly, in contrast to Dendra2 ${ }_{\mathrm{T}_{69} \mathrm{~A}^{-}}$ Ldcl, mGeosM-Ldcl assembled into regular symmetric non-native higher-order oligomers, with a dramatically altered quaternary structure (Figure 1B, Supplementary Figure 1). These oligomers were built of three Ldcl tetramers, bridged together by additional densities. Noteworthy, mEos2, the fluorescent protein from which mGeosM was derived, crystallises as a tetramer (PDB ID: 3S05) that can be straightforwardly fitted into the Ldcl-bridging densities (Figure 1B). This illustrates that despite the fact that mGeosM was designed as a monomeric FP (with the first " $m$ " explicitly standing for monomeric), some residual oligomeric tendency is still maintained. This propensity of mGeosM to oligomerise when bound to Ldcl may be driven by avidity effects - as Ldcl dimers begin to assemble into a decamer, the local concentration of mGeosM increases to such a point that oligomerisation becomes energetically favourable, despite the apparent monomer behaviour of mGeosM in gel filtration (26). This is also in line with the known propensity of mEos2, from which mGeosM is derived, to form tetramers at high concentration (34). To conclude, both $\mathrm{N}$-terminal fusions induced non-native assembly of Ldcl, and therefore neither were appropriate for determining the native localisation of this enzyme in the cell.

As expected, C-terminal Ldcl fluorescent fusions formed decamers with protruding densities that can be attributed to flexibly attached FPs (Figure 1B, Supplementary Figure 1). Nevertheless, despite the long flexible linker between $\mathrm{Ldcl}$ and the FP, these constructs were unable to interact with RavA as shown by Bio-Layer Interferometry (BLI) binding studies (see Methods and Supplementary Figure 1 for details). This means that the functionality of these fusions cannot be considered as entirely retained. Thus, none of the four fusions were suitable for an in-depth imaging analysis under conditions of native Ldcl expression upon acid stress that we planned to undertake. Altogether, we demonstrated that structural integrity and unaltered interaction with known partners are useful read-outs for functional preservation. Based on this result we propose that, when feasible, purification and structure-function analysis of FP fusions should be performed prior to interpretation of the protein localisation inferred from observation of FP fusions by optical methods. This workflow can be used for example in cases where chromosomal manipulation for assessment of intact function is difficult and/or the phenotype is condition-dependent or unclear, whereas basic in vitro biochemical and ns-EM characterisation are efficiently set up. 


\section{Immunofluorescence of Ldcl in E. coli under acid stress reveals its supramolecular organisation}

Because none of the FP fusions possessed the properties of native Ldcl, we decided to turn to STORM imaging of the endogenous enzyme. One of the drawbacks of STORM is the requirement for exogenous labelling and therefore the fixation and permeabilisation of cells. These procedures are notoriously known to potentially affect cell morphology, and therefore, when possible, the usage of live cell imaging with FP fusions (as for example in PALM), or observation of unfixed samples under cryogenic conditions would be ideal. Moreover, fixation, permeabilisation and specific exogenous labelling in bacteria present unique challenges because of the complex, often multi-layered cell wall $(35,36)$, A significant advantage of STORM however is the possibility of direct imaging of the wild-type (WT) protein, which should circumvent the dangers associated with FP fusions described above. The three prerequisites for imaging of WT systems by immunobased labelling in general and of Ldcl in particular are (i) availability of an antibody or nanobody (antibody fragment derived from heavy-chain-only camelid antibody) coupled to an organic fluorescent dye and directed towards the native protein, (ii) precise knowledge of endogenous expression conditions, (iii) validation of an efficient permeabilisation and immunolabelling technique that enables the antibody/nanobody to enter the cell and specifically target the protein of interest without altering the native organization of the cell ultrastructures.

As a first step towards STORM imaging of endogenous Ldcl, we probed an anti-Ldcl nanobody, hereafter called anti-Ldcl-Nb. The complex between purified $E$. coli Ldcl and the nanobody was purified by size exclusion chromatography (see Methods), and imaged by ns-EM. The resulting 3D map of the Ldcl/anti-Ldcl-Nb complex demonstrates binding of anti-Ldcl-Nb to each Ldcl monomer in the decamer and reveals the location of the interaction site which is clearly distinct from the RavA binding site (Figure 2A, Supplementary Figure 2). This nanobody was thus identified as a suitable labelling agent for Ldcl imaging in $E$. coli cells, and labelled with the fluorescent dye AlexaFluor647 (AF647) or AlexaFluor488 (AF488) (see Methods).

Consistently with published data, Ldcl expression could be induced by a pH shift experiment (i.e. transfer of bacteria from pH 7.0 into a pH 4.6 growth medium) in the presence of lysine under oxygen-limiting conditions (Supplementary Figure 3A). While the WT strain grew well under these conditions and efficiently buffered the extracellular medium up to $\mathrm{pH} 6.2$ in approximately 1.5 to two hours (Supplementary Figure $3 \mathrm{~A}$ ) concomitantly with the increase in the level of $\mathrm{Ldcl}$ expression (Supplementary Figure 3B, C), the growth and the acid stress response of the $\Delta l d c l$ mutant strain were severely impaired (Supplementary Figure $3 \mathrm{~A}$ ). Since the peak of Ldcl expression by the wild type cells was achieved between one and two hours after exposure to acid stress (Supplementary Figure 3B, C), a time point of 90 minutes was chosen for the subsequent labelling and imaging experiments. The specificity and performance of anti-Ldcl- $\mathrm{Nb}$ in immunofluorescence labelling of permeabilised $E$. coli cells were characterised by flow cytometry and wide-field fluorescence imaging (Supplementary Figure 3D, E). Both techniques demonstrated that in the absence of Ldcl expression no specific fluorescence is seen, confirming the suitability of anti-Ldcl-Nb for immunofluorescence studies. Thus, the above-mentioned prerequisites for immuno-based imaging of cellular Ldcl have been fulfilled. Noteworthy, the Ldcl expression profile 
highlighted a considerable asset of the usage of STORM instead of PALM for visualisation of the endogenously expressed Ldcl: indeed, the transient nature of the expression and the necessity of work under oxygen-limiting conditions may have created difficulties due to the longer maturation time of the FPs under these conditions.

Initial characterisation of the cellular distribution of Ldcl 90 minutes after exposure of $E$. coli cells to acid stress was carried out by wide-field fluorescence imaging. Based on these images, it appeared that natively expressed Ldcl did not display a homogeneous cytoplasmic distribution but rather showed a patchy localisation pattern (Figure 2B). 3D STORM imaging subsequently provided a more detailed view of this patchy distribution. As shown in Figure 3, Supplementary Figure 4, and Supplementary Movies 1-5, the labelling density was lower in the centre of the bacterium. This indicates a propensity for Ldcl to cluster near the cell periphery, towards the inner membrane and the cell poles, rather than being distributed homogeneously through the volume. Recent investigations argue that, in most cases, in vivo clustering of metabolic enzymes corresponds to their polymerised states, and represents an efficient means of regulation of enzymatic activity and metabolic homeostasis in response to a stimulus (1). Thus, structure determination of these polymers is a crucial step towards the understanding of regulation mechanisms. We previously documented that in vitro at $\mathrm{pH}$ below 5 and high concentration, Ldcl decamers indeed tend to stack (6). However, at pH 5.7, optimal for Ldcl expression and enzymatic activity and likely corresponding to the internal $\mathrm{pH}$ upon acid stress, the oligomeric state of Ldcl has not yet been addressed by EM. Supposing that the high local concentration of Ldcl, clustered in patches in the $E$. coli cell under acid stress conditions, is likely to induce enzyme polymerisation via stack formation, we next examined the Ldcl assembly state at $\mathrm{pH} 5.7$ by cryo-EM and discovered that it does indeed form straight polymers (Figure 4).

\section{Structural determinants of Ldcl stack formation revealed by cryo-EM}

In order to understand the molecular determinants of $\mathrm{Ldcl}$ polymerisation at low $\mathrm{pH}$ and to provide a framework for the analysis of Ldcl function under acid stress, we solved the 3D structures of the Ldcl decamer at $\mathrm{pH} 7.0$ and of Ldcl stacks at pH 5.7 by cryo-EM (See Methods, Figure 4, Supplementary Figures 5-6, Supplementary Table 1 ). The $2.8 \AA$ resolution structure of the $\mathrm{Ldcl}$ decamer at pH 7.0 (Figure 4A) is extremely similar (Supplementary Table $2 \& 3$ ) to the Ldcl crystal structure solved at pH 8.5 in an inhibited ppGpp-bound state (6). However, contrary to $\mathrm{pH} 7.0$ and even $\mathrm{pH} 6.2$ where $\mathrm{Ldcl}$ is still predominantly decameric (10), Ldcl forms straight rigid filaments on the cryo-EM grid at $\mathrm{pH} 5.7$, which corresponds to the $\mathrm{pH}$ of maximum Ldcl enzymatic activity (Figure 4B). The structure of a three-decamer stack was solved to a resolution of $3.3 \AA$, revealing the structural details of acid stress-induced Ldcl polymerisation (Figure 4C). Ldcl decamers stack tightly on top of one other, with negligible rotation between decamers along the stack. Each dimer fits snugly in the inter-dimer groove of decamers above and below.

A comparison of $\mathrm{Ldcl}$ decamer structures taken from the Ldcl stack cryo-EM map (at $\mathrm{pH}$ 5.7), the Ldcl decamer cryo-EM map (at $\mathrm{pH}$ 7.0) and the crystal structure of decameric Ldcl crystallised with bound ppGpp at pH 8.5 (PDB ID: 3N75) reveals some remarkable differences between the stack structure and the two decamer structures (Figure 4D, E, Supplementary Table 2 \& 3). While the three structures do not show any major differences at the monomer level, a 
structural alignment of an Ldcl dimer extracted from the Ldcl decamer structures with a dimer extracted from the Ldcl stack structure uncovers a rigid body-like rotation between monomers around a hinge region located at the monomer-to-monomer interface (Supplementary Table 2 \& 3 , Figure $4 \mathrm{DE}$ ). This rotation results in a $5^{\circ}$ tilt when comparing the $\mathrm{N}$-terminal wing-domains in Ldcl dimers, and an overall slightly decreased diameter of the central cavity inside the stacked Ldcl rings (Figure 4D, E), which may contribute to the tight packing of each dimer into the grooves of an opposing decamer in the stack.

A careful examination of the Ldcl stack structure shows that two major inter-decamer interfaces situated at a two-fold symmetry axis perpendicular to the stack direction contribute to stack formation (Figure 5A). In particular, the first interface (Figure 5B) is formed between residues K422, D460, R468, D470, and E482 situated in the ppGpp-binding domain (amino acids 418-564), and residues N314, D316, and G352 from the PLP-binding domain (amino acids 184-417). D460 from one decamer makes an electrostatic interaction with K422' of a neighbouring decamer in the stack. R468 is sandwiched between D316' and E344' from a neighbouring decamer, and makes electrostatic interactions through its $\eta 1$ and $\eta 2$ nitrogen atoms with D316'. In addition, D470 interacts with the backbone of G352', and E482 forms hydrogen bonds with N314'. The second interface (Figure 5B) is formed between residue N94 of the wing domain (amino acids 1-130) of one set of opposing dimers, and a stretch of four residues in the ppGpp-binding domain - T444, E445, S446 and D447 - at the end of helix a16 from a second set of opposing dimers. The wing domain residue N94 makes hydrogen bonds with E445' of an opposing dimer. A second charged residue, D447, interacts with the backbone of T444', and is held in place by R97 from the wing domain of a neighbouring dimer in the same decamer.

Considering that the Ldcl polymerisation is induced by acid stress, we wondered which residues in the interface would be sensitive to $\mathrm{pH}$ changes. Surprisingly, most of the side chains involved in the inter-decamer interface are charged arginine (pKa 13), aspartate and glutamate residues (pKa of 4 and 3 respectively), which do not change protonation state in the $\mathrm{pH}$ window relevant for Ldcl activity ( $\mathrm{pH} \mathrm{5-7)} \mathrm{(37).} \mathrm{Nonetheless,} \mathrm{other} \mathrm{residues,} \mathrm{situated} \mathrm{outside} \mathrm{the} \mathrm{interaction}$ interfaces, may drive stack formation through $\mathrm{pH}$-dependent interactions that would in turn lead to the observed inter-monomer rotation and the associated constriction of the Ldcl central cavity, coupled to the alignment of complementary contacts at interfaces one and two (Figure 4D, E). We note for example that $\mathrm{H} 694$ should be protonated in the Ldcl stack structure at $\mathrm{pH} 5.7$ but deprotonated in the two ring structures at $\mathrm{pH} 7.0$ and $\mathrm{pH} 8.5$, and that an electrostatic interaction between $\mathrm{H} 694$ and D192 situated in the linker region is present in the stack structure only (Figure $5 E)$.

To validate the observed interactions at the inter-decamer interface, and to assess the individual importance of key residues involved in Ldcl stack formation, we constructed four Ldcl point mutants (R468E, R97E, H694A and H694N), two double mutants (R97E/R468E and E445A/D447A) and one triple mutant (E445A/D447A/R468E). The mutants were purified following the protocol for wild-type Ldcl (see Methods), diluted into a buffer at pH 5.7 and observed by nsEM (Figure 6, Supplementary Figure 7). Although the grid preparation procedure for ns-EM yields stacks that are shorter and more curved and distorted when compared to the cryo-EM data (Figure $4 \mathrm{~B}$, Figure 6), our previous observations of the five paralogous $E$. coli amino acid decarboxylases 
justify the validity of this approach for a qualitative comparative analysis $(6,37)$. Ns-EM images make immediately apparent that the E445A/D447A double mutant does not have a significantly altered capability of stack formation at $\mathrm{pH} 5.7$ when compared to WT Ldcl, whereas the single R468E mutation is sufficient to completely abolish stack formation. The R97E mutant has a moderate destabilising effect and displays fewer and smaller stacks then the WT Ldcl. Consistently with the major role of R468 in the Ldcl stack formation, the R97E/R468E double mutant and $\mathrm{E} 445 \mathrm{~A} / \mathrm{D} 447 \mathrm{~A} / \mathrm{R} 468 \mathrm{E}$ triple mutant exclusively occur as decamers at low $\mathrm{pH}$. Altogether, these results reveal that $\mathrm{R} 468$ is one of the key determinants of Ldcl stack formation. Finally, similarly to the R97E mutant, a modest destabilisation of stack formation is observed for the two histidine mutants, H694A and H694N, favouring our hypothesis that H694 may have an influence on the propensity of Ldcl polymerisation at low pH (Figure 6, Supplementary Figure 7).

\section{Discussion}

Our synergistic approach, combining several in vitro techniques including biochemical characterisation of purified fluorescent protein fusions, ns-EM observation of mutants, low resolution ns-EM reconstruction and high resolution cryo-EM analysis, with in vivo flow cytometry, wide-field and 3D-STORM imaging, provides insights into the supramolecular Ldcl assembly upon acid stress. This work adds to the very few known examples of regular polymerisation as means of regulation of enzymes involved in amino acid metabolism in bacteria. The cryo-EM structure of the Ldcl stacks presented here offers a molecular framework for future investigation of the role of the Ldcl polymerisation in the acid stress response.

The high resolution cryo-EM structures of the stack at $\mathrm{pH} 5.7$ and of the decamer at $\mathrm{pH} 7.0$ complement the previously solved crystal structure of the ppGpp-bound Ldcl decamer obtained at $\mathrm{pH}$ 8.5. Previous serendipitous co-crystallisation of $\mathrm{Ldcl}$ with the nutrient stress response alarmone ppGpp led to assessment of the effect of ppGpp on Ldcl activity, and to a proposal that ppGpp would act as an Ldcl inhibitor that prevents excessive lysine consumption upon nutrient limitation during acid stress (6). In addition, a ppGpp-dependent disassembly of the Ldcl stacks had been previously observed but could not be structurally explained since the ppGpp binding site is situated between two neighbouring dimers inside the Ldcl ring (6). Our cryo-EM structures show that one of the residues involved both in ppGpp binding and in the stack formation is arginine 97 (R97). In the crystal structure of ppGpp-bound Ldcl, R97 makes a stacking interaction with the guanosine imidazole ring of ppGpp, while in Ldcl stacks R97 is involved in a key interaction at the second interface (Figure 5D), where it locks D447 in a conformation allowing interactions between helices a16 from opposing Ldcl decamers. Binding of ppGpp to Ldcl interferes with the R97-D447 interaction, thereby most likely prohibiting correct positioning of D447 at the tip of helix a16, and resulting in a disruption of the second stack interface, leading to a moderate yet notable stack destabilisation (Figure 6, Supplementary Figure 7). Furthermore, our cryo-EM structure of ppGppfree $\mathrm{Ldcl}$ decamers at neutral $\mathrm{pH}$ enables to rule out the effect of ppGpp on the differences observed between the Ldcl stack structure at pH 5.7 and the ppGpp-Ldcl crystal structure at $\mathrm{pH}$ 8.5. Indeed, despite the absence of ppGpp, R97 is still oriented towards the ppGpp binding site and away from the inter-decamer interface in the $\mathrm{pH} 7.0$ decamer cryo-EM map (Figure 5D). This suggests that the conformational changes in Ldcl driving stack formation are mostly driven by low 
$\mathrm{pH}$ and not by the absence of ppGpp, although the D192 and H694 hinge residues are similarly far apart in the $\mathrm{pH} 8.5$ and $\mathrm{pH} 7.0$ structures (Figure 5E). Our current work provides a solid experimental and structural basis for a future closer evaluation of the hypothesised role of ppGpp in Ldcl regulation in vitro and in vivo (6)

Although the optical imaging experiments performed in this study do not allow quantitative evaluation of the nature of the clusters of endogenous Ldcl at the bacterial periphery, they would presumably correspond to Ldcl assembled into stacks. Possible reasons for such assembly may be to provide an efficient way to locally increase the Ldcl concentration and to enhance its activity. But why would Ldcl, an apparently highly soluble protein, be driven towards the inner membrane? What would be the advantage for acid-stressed E. coli cells to increase the concentration of Ldcl via stack formation in these particular peripheral locations? Localisation of proteins to specific sites in the bacterial membrane was shown to be generally driven by chemical factors such as the phospholipid composition of the lipid microdomains, and by physical factors such as the degree of local curvature or the electric potential of the membrane (38). An attractive hypothesis would be that as an acid stress response protein performing a proton-consuming enzymatic reaction, Ldcl may be attracted to proton sinks formed by anionic phospholipids which compartmentalise oxidative phosphorylation (OXPHOS) complexes for efficient functioning in bacterial respiration and adaptation to environmental changes. Indeed, OXPHOS complexes were often described to be unevenly distributed in the membrane in the form of mobile patches (39-43), providing evidence for highly dynamic and spatially organised bioenergetic membranes in E. coli cells (43). In addition, certain bacterial flottilins, which are essential scaffold proteins of the functional membrane microdomains, equivalent to the lipid rafts of eukaryotic cells, also show a patchy distribution and were shown to interact with specific OXPHOS complexes $(43,44)$. In this regard, two different lines of evidence would be interesting to note. First, Ldcl was described to co-purify with a partially assembled Complex I (45), whereas the Ldcl-binding partner RavA, as well as ViaA, the second protein expressed from the ravAviaA operon and which also interacts with RavA, were shown to interact with both Complex I and fumarate reductase $(32,33)$. Second, the other E. coli PLPdependent lysine decarboxylase LdcC, exercising the role of cadaverine biosynthesis irrespectively of acid stress (46), neither binds RavA nor forms stacks $(29,37)$, in spite of its $69 \%$ of identity with Ldcl. It is interesting to note that in LdcC the key stack-forming residue R468 has been substituted for an alanine while the interacting D316 has been preserved.

Our STORM images suggest that individual patches have a peripheral distribution with a long-range stripy or pseudo-helical organisation (Figure 3, Supplementary Figure 4, Supplementary Movies 1-5). While similar distributions have been documented for bacterial cytoskeletal, cell division, chromosome partitioning, RNA degradation and secretion machineries, an eventual impact of labelling on these distributions, demonstrated specifically for the YFP-MreB (47), warrants caution in their interpretation $(13,15)$. Here however, we observed endogenous wild-type $\mathrm{Ldcl}$ in cells fixed prior to their permeabilisation and labelled with anti-Ldcl-Nb, which means that the resulting pattern is likely real. In addition, examination of some published images of OXPHOS patches (for example Llorente-Garcia et al., 2014) also hints to a similar organisation. Excitingly, anionic phospholipid-specific dyes and fluorescently-labelled antibiotics specific for nascent peptidoglycan synthesis upon cell elongation were also proposed to be distributed on 
helical or stripe patterns $(38,48-51)$. It is therefore tempting to imagine that inside the bacterial cell, Ldcl has a tendency to follow a general path upon polymerisation governed by the underlying chiral patterns in the cell envelope (52).

Finally, from the methodological view, our work convincingly illustrates that different FP fusion constructs can share the same cellular distribution in spite of a completely different structure, necessitating caution when inferring intact function from the preservation of the protein localisation inside the cell. Our findings emphasize the importance of characterising FP fusions using both biochemical and structural techniques, such as ns-EM, to ensure that the FP tag disrupts neither structure nor function of the target protein.

\section{Methods}

\section{Expression constructs}

For fluorescence studies, several FP fusion constructs were generated starting from an available plasmid containing the coding sequence of Ldcl (Uniprot entry POA9H3), cloned in the $\mathrm{pET} 22 \mathrm{~b}(+)$ vector with a C-terminal 6xHis-tag (6). All constructs were generated using the Gibson cloning strategy and verified by sequencing analysis. The Gibson assembly was performed using 0,4U T5 exonuclease, 2,5U Phusion polymerase and 400U Taq ligase (New England Biolabs) in 1X ISO buffer (100mM Tris- $\mathrm{HCl} \mathrm{pH} 7,5,10 \mathrm{mM} \mathrm{MgCl}$, $0.8 \mathrm{mM}$ dNTP mix, 10mM DTT, $50 \mathrm{mg}$ PEG-8000, $1 \mathrm{mM}$ NAD). $7.5 \mu \mathrm{L}$ of the GIBSON Master Mix was mixed with $2.5 \mu \mathrm{L}$ DNA, containing circa $100 \mathrm{ng}$ of vector. The mix was incubated for $60 \mathrm{~min}$ at $50^{\circ} \mathrm{C}$. Transformations were performed in Top10 competent bacteria (One Shot ${ }^{\mathrm{TM}}$ TOP10 Chemically Competent E. coli, Invitrogen) and selected using $100 \mu \mathrm{g} / \mathrm{mL}$ ampicillin or $50 \mu \mathrm{g} / \mathrm{mL}$ kanamycin sulphate (Euromedex). Agarose Gel purification and DNA plasmid extraction kits were purchased from Macherey-Nagel.

Dendra2 ${ }_{\text {T69A }}-\mathrm{Ldcl}$ and mGeosM-Ldcl were both cloned in the pET-TEV vector containing an $\mathrm{N}$-terminal 6xHis-tag, and a TEV cleavage site between Denda2 ${ }_{\text {T69А }}$ or mGeosM and the Ldcl gene. Ldcl-Dendra2 ${ }_{\text {T69A }}$ and Ldcl-mGeosM were both cloned in the pET22b(+) vector with Ldcl followed by either Dendra2 ${ }_{\text {T69A }}$ or mGeosM containing an uncleavable C-terminal 6 XHis-tag.

The anti-Ldcl-Nb was obtained from the nanobody generation platform of the AFMB laboratory (Marseille, France). A llama (Llama glama) was immunized with the purified wild type Ldcl. Lymphocytes were isolated from blood samples, a nanobody phage display library was generated, and enrichment of antigen-specific clones was performed using standard procedures $(53,54)$. Sequences of three positive clones were subcloned in the pHEN6 vector containing the pelB leader sequence from Erwinia carotovora for secretion into the periplasm, and a C-terminal 6xHis-tag (kindly provided by Dr. Aline Desmyter, AFMB Marseille). One of the clones was used for the present study to yield anti-Ldcl-Nb.

Plasmids, primers and cloning strategy are summarised in Supplementary Table 4.

\section{Protein purification}

Ldcl-FP fusions and Ldcl mutants were expressed in BL21(DE3) cells grown in LB medium supplemented with $100 \mu \mathrm{g} / \mathrm{mL}$ ampicillin or $50 \mu \mathrm{g} / \mathrm{mL}$ kanamycin sulphate. Protein expression was induced using $40 \mu \mathrm{M}$ IPTG (Euromedex) and carried out overnight at $18^{\circ} \mathrm{C}$. The Ldcl-FP fusions 
and Ldcl mutants were purified as previously described for wild-type $\operatorname{Ldcl}(28,29,37)$, in a final buffer containing $25 \mathrm{mM}$ Tris (pH 7.5), $0.3 \mathrm{M} \mathrm{NaCl}, 5 \%$ glycerol, $1 \mathrm{mM}$ DTT and $0.1 \mathrm{mM}$ PLP.

The anti-Ldcl-Nb was expressed in E. coli WK6 cells following the protocol described by (53), and purified with immobilised metal affinity chromatography (IMAC, using a Ni-NTA column) followed by Size exclusion chromatography (SEC) using a a superdex 75 Increase 10/300GL column (GE-Healthcare) equilibrated with a buffer containing $25 \mathrm{mM}$ Tris $\mathrm{pH} 7.4$ and $0.3 \mathrm{M} \mathrm{NaCl}$.

In order to characterise the Ldcl complex with anti-Ldcl-Nb, the two proteins were mixed at a 1:5 molar ratio and submitted to size exclusion chromatography as carried out for Ldcl alone but without DTT in the buffer. The top of the peak was taken for subsequent ns-EM analysis.

\section{Ns-EM on Ldcl-FP fusions, Ldcl/anti-Ldcl-Nb and Ldcl mutants}

FP fusion samples after gel filtration were diluted to a concentration of approximately 0.025 $\mathrm{mg} / \mathrm{mL}$. $3 \mu \mathrm{L}$ was applied to the clean side of carbon on a carbon-mica interface and stained with 2\% uranyl acetate (mGeosM-Ldcl, Ldcl-mGeosM, Ldcl-Dendra2 ${ }_{\text {T69A }}$, Ldcl/anti-Ldcl-Nb, Ldcl mutants) or $2 \%$ sodium silicotungstate (Dendra2 ${ }_{\text {T69A }}-\mathrm{Ldcl}$ ). Images were collected on a $120 \mathrm{kV}$ Tecnai T12 microscope with an Orius 1000 camera (Gatan) or on a 200 kV Tecnai F20 electron microscope with either a OneView camera (Gatan) or a Ceta camera (Thermo Scientific). All images were collected with a defocus range of approximately $-1.0 \mu \mathrm{m}$ to $-2.0 \mu \mathrm{m}$ and with pixel sizes between $2.29 \AA /$ pixel and $3.42 \AA ̊ / p i x e l$.

\section{Image processing - Ldcl-FP and Ldcl/anti-Ldcl-Nb}

36 micrographs of Dendra2 ${ }_{\text {T69A }}$-Ldcl with a pixel size of $2.73 \AA /$ pixel, 368 micrographs of mGeosM-Ldcl with a pixel size of $2.29 \AA$ Åpixel, 23 micrographs of Ldcl-Dendra2 ${ }_{\text {T69A }}, 92$ micrographs of Ldcl-mGeosM with a pixel size of $3.42 \AA /$ pixel, and 124 micrographs of Ldcl/antiLdcl-Nb with a pixel size of $2.82 \AA /$ pixel were used for image analysis.

CTF estimation was performed with CTFFIND3 (55). Semi-automatic particle selection was

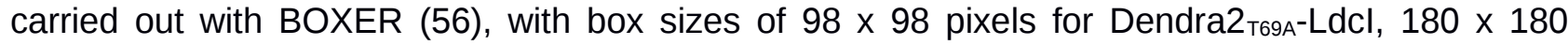
pixels for mGeosM-Ldcl, $128 \times 128$ pixels for Ldcl-Dendra2 ${ }_{\text {T69A A }}$ and Ldcl-mGeosM, and $112 \times 112$ pixels for Ldcl/anti-Ldcl-Nb respectively. Particle extraction followed by several rounds of cleaning by 2D classification in RELION-1.4 (57), resulted in the following number of particles for each dataset: Dendra2 ${ }_{\text {T69A }}-\mathrm{Ldcl}=7140, \mathrm{mGeosM}-\mathrm{Ldcl}=5514$, Ldcl-Dendra2 $_{\text {T69A }}=832$, Ldcl-mGeosM $=$ 12,211 and Ldcl/anti-Ldcl-Nb = 14,075.

For Dendra2 ${ }_{\text {T69A }}-\mathrm{Ldcl}$, initial model generation was carried out in RELION-2.1 (58) without any symmetry applied. For mGeosM-Ldcl, initial model generation was carried out in RELION-2.1 with either C1, C3 or D3 symmetry applied. The results of all three calculations being very similar, the model with applied D3 symmetry was selected. For Ldcl-Dendra2 ${ }_{\text {T69A }}$, Ldcl-mGeosM and Ldcl/anti-Ldcl-Nb, the previously-determined Ldcl decamer structure (PBD ID: 3N75) was filtered to $60 \AA$ and used as an initial model for 3D refinement.

3D refinement was carried out for each dataset with applied C2 symmetry for Dendra ${ }_{\text {T69A }^{-}}$ Ldcl, D3 symmetry for mGeosM-Ldcl, and D5 symmetry for Ldcl-Dendra2 ${ }_{\text {T69A }}$, Ldcl-mGeosM and Ldcl/anti-Ldcl-Nb. Rigid body fitting of Ldcl (PBD ID: 3N75), mEos2 (PDB ID: 3S05) and Dendra2 (PDB ID: 2VZX) crystal structures was then carried out in Chimera (59) for the five datasets. 
462

\section{Cryo-EM on Ldcl stacks (pH 5.7)}

Wild-type Ldcl was purified as previously described (10) from an E. coli strain impaired in the production of ppGpp (MG1655 $\Delta$ relA $\Delta s p o T$ ) in order to avoid any serendipitous ppGpp binding. Purified Ldcl was diluted to a final concentration of approximately $0.25 \mathrm{mg} / \mathrm{mL}$ in a buffer containing $25 \mathrm{mM}$ MES (pH 5.7), $0.3 \mathrm{M} \mathrm{NaCl}, 5 \%$ glycerol, $1 \mathrm{mM}$ DTT and $0.1 \mathrm{mM} \mathrm{PLP.} 3 \mu \mathrm{L}$ of the sample was applied to a glow-discharged R2/1 300 mesh holey carbon copper grid (Quantifoil Micro Tools $\mathrm{GmbH}$ ) and plunge-frozen in liquid ethane using a Vitrobot Mark IV (FEI) operated at $100 \%$ humidity. Datasets were recorded at the European Synchrotron Radiation Facility (ESRF) in Grenoble, France (60), on a Titan Krios microscope (Thermo Scientific) equipped with a BioQuantum LS/967 energy filter (Gatan) and a K2 summit direct electron detector (Gatan) operated in counting mode. A total of 2564 movies of 30 frames were collected with a total exposure of $6 \mathrm{~s}$, total dose of $29.3 \mathrm{e} / \AA^{2}$ and a slit width of $20 \mathrm{eV}$ for the energy filter. All movies were collected at a magnification of $130,000 x$, corresponding to a pixel size of $1.052 \AA /$ pixel at the specimen level. A summary of cryo-EM data collection parameters can be found in Supplementary Table 1.

\section{Image Processing - Ldcl stacks (pH 5.7)}

Motion correction and dose-weighting of the recorded movies were performed using MotionCor2 (61). CTF parameters were determined on the aligned and dose-weighted sums using CTFFIND4 (62). After manual inspection of the dose-weighted sums, the best 558 (21.7\%) micrographs were selected for further processing. Ldcl stacks were manually picked using e2helixboxer in EMAN2 (63). A total of 15,165 Ldcl-stack particles were extracted in RELION-3.0 (64) with an extract size of 320 pixels, resulting in boxes containing three Ldcl decamers, and with the --helix option with an outer diameter of 160 pixels and a helical rise of 77 pixels. After particle extraction, per-particle CTF correction was performed using Gctf (65). Extracted particles were subjected to 2D classification in RELION-3.0, resulting in a cleaned dataset containing 15,157 particles. Initial 3D refinement with imposed D5 symmetry was carried out in RELION-3.0, using an initial model generated by manually stacking three Ldcl decamers (PDB ID: 3N75) in Chimera (59) and low-pass filtering the resulting Ldcl-stack to $40 \AA$. The resulting $4.3 \AA$ resolution 3D reconstruction, along with the cleaned particle stack, was subsequently imported into CryoSPARC (66). A final homogeneous 3D refinement in CryoSPARC, using a dynamic mask and imposing D5 symmetry, resulted in a map with a resolution of $3.28 \AA$ based on the 0.143 gold-standard Fourier shell correlation (FSC) criterion (67). The final map was sharpened using a B-factor of $-96 \AA^{2}$. A local resolution estimation of the final 3D reconstruction was calculated in RELION-3.0. A summary of cryo-EM data collection parameters and image processing steps for Ldcl stacks can be found in Supplementary Table 1 and Supplementary Figure 4, with local resolution and FSC curves shown in Supplementary Figure 5.

\section{Cryo-EM on Ldcl decamers (pH 7.0)}

Purified Ldcl was dialysed into a buffer at $\mathrm{pH} 7.0$ and diluted to a concentration of 0.25 $\mathrm{mg} / \mathrm{mL}$. $3 \mu \mathrm{L}$ of diluted sample was applied to a glow-discharged (R2/1 300 mesh holey carbon 
copper grid (Quantifoil Micro Tools $\mathrm{GmbH}$ ) and plunge-frozen in liquid ethane using a Vitrobot Mark IV (FEI) operated at $100 \%$ humidity. Images were recorded on a Glacios microscope (Thermo Scientific) equipped with a Falcon II direct electron detector (Thermo Scientific). A total of 2772 movies of 29 frames were collected with a total exposure of $1.5 \mathrm{~s}$ and a total dose of $45 \mathrm{e} / \AA^{2}$. All movies were collected at a magnification of $116,086 x$, corresponding to a pixel size of 1.206 $\AA /$ pixel at the specimen level.

\section{Image Processing - Ldcl decamers (pH 7.0)}

Motion correction was carried out using patch motion correction in CryoSPARC, discarding the first two frames. Initial CTF estimation was then carried out on summed frames using CTFFIND4. A subset of 600 micrographs were subjected to automatic picking using the blob picker in CryoSPARC, resulting in $\sim 238,000$ picked particles. Particles were then extracted with a box size of $256 \times 256$ and subjected to 2D classification. Particles from the best classes showing clear secondary structural features for Ldcl were selected for homogeneous refinement (with applied D5 symmetry) against EMD-3204 low-pass filtered to $30 \AA$, resulting in a reconstruction with a resolution of $4.2 \AA$ (FSC $=0.143$ ). This reconstruction was then used to create templates for picking the entire dataset using the template picker in CryoSPARC, after filtering to $12 \AA . \sim 796,000$ particles were extracted and subjected to $2 \mathrm{D}$ classification, and the best $\sim 428,000$ particles were subjected to heterogeneous refinement with applied D5 symmetry against the $4.2 \AA$ map, resulting in one higher-resolution class corresponding to $\sim 229,000$ particles. These particles were subjected to homogeneous refinement with applied D5 symmetry, resulting in a map with a resolution of 2.76 $\AA(F S C=0.143)$ which was then sharpened with a B-factor of $-173 \AA^{2}$. A summary of cryo-EM data collection parameters and image processing steps for $\mathrm{Ldcl}$ decamers can be found in Supplementary Table 1 and Supplementary Figure 4, with local resolution and FSC curves shown in Supplementary Figure 5.

\section{Fitting of structures and refinement}

For fitting of atomic models in the 3D reconstructions of Ldcl at pH 5.7 (stack) or pH 7.0 (decamer), two (for the stack) or one copy (for the decamer) of the Ldcl X-ray crystal structure (PDB ID: 3N75) were first rigid-body fitted in the corresponding 3D reconstructions using Chimera. Refinement was performed using the Phenix software package (68) and was identical for both 3D reconstructions. A first round of real space refinement was carried out with enabled rigid-body, global minimization, local grid search and ADP refinement parameters, and imposing rotamer, Ramachandran, NCS and reference model (PDB ID: 3N75) restraints. A final round of real space refinement was then performed using the same settings, but without rigid body refinement and without applying reference restrains, setting the 'nonbonded weight' parameter to 4000 and disabling 'local_grid_search'. A summary of refinement and model validation statistics can be found in Supplementary Table 1.

\section{pH shift experiment}

Stationary phase cultures, which were grown overnight from single colonies in LB medium, were diluted to OD600 0.01 and re-grown at $37^{\circ} \mathrm{C}$ within approximately $1 \mathrm{~h} 45 \mathrm{~min}$ in fresh LB 
medium to an OD600 of 0.1 . From this culture, $14 \mathrm{~mL}$ were transferred to $15 \mathrm{~mL}$ falcon tubes and pelleted by centrifugation at RT for 5 min. The supernatant was decanted, whereby systematically around $200 \mu \mathrm{L}$ LB remained in the falcon tube. The pellet of bacteria was resuspended and afterwards, LB-4. 6 containing $30 \mathrm{mM}$ L-lysine was added to the cells up to $14 \mathrm{~mL}$. To prepare LB4.6 medium, LB powder (Sigma-Aldrich) was completely dissolved in distilled water by stirring for $30 \mathrm{~min}$. The $\mathrm{pH}$ of 4.6 was then adjusted using $\mathrm{HCl}$. After autoclaving, sterile filtered L-lysine was added to LB-4.6. For this, L-lysine was dissolved in an aliquot of LB-4.6, sterile filtered and mixed with the remaining LB-4.6. $30 \mathrm{mM}$ L-lysine were used. In order to grow the culture under oxygenlimiting conditions, the lid of the falcon tube was closed and the tubes were placed at $37^{\circ} \mathrm{C}$ on a shaker $(150 \mathrm{rpm})$. After defined time-points, aliquots were taken for OD600 measurement, $\mathrm{pH}$ measurement, SDS-PAGE or immunofluorescence. For each time-point, a new tube was opened and not reused further.

\section{Western Blotting}

SDS-PAGE was performed with a Biorad electrophoresis chamber using standard $12 \%$ reducing SDS-PAGE gels. Proteins were transferred to a nitrocellulose membrane (Biorad) using a TransBlot Turbo Transfer System (Biorad). The membrane was blocked for 1 h using 5\% BSA in TBS supplemented with $0.1 \%$ Tween (TBS-T). Afterwards, the membrane was incubated for $1 \mathrm{~h}$ with an anti-Ldcl antibody (Qalam-Antibodies) in BSA/TBS-T (1:5000). The membrane was subsequently washed $3 \times 10 \mathrm{~min}$ in TBS-T and incubated for $1 \mathrm{~h}$ with HRP-coupled anti-rabbit antibody (1:10000 in BSA/TBS-T). Finally the membrane was washed $3 \times 10 \mathrm{~min}$ in TBS-T. The membrane was rinsed once with TBS, prior to detection of antibody-labelled proteins using ECL reagent (GE Heathcare). Incubation and detection of antibody was performed at $25^{\circ} \mathrm{C}$.

\section{Nanobody labelling}

For the labelling reaction, $50 \mu \mathrm{L}$ nanobody (i.e. $200 \mu \mathrm{g}$ ) was pipetted into a $1.5 \mathrm{~mL}$ tube, placed on ice and supplemented with $5.5 \mu \mathrm{L}$ of $1 \mathrm{M}$ bicarbonate buffer at pH 8.3. $100 \mu \mathrm{g}$ of Alexa647 or Alexa-488 NHS ester dye (Life Technologies, A37573 and A20000) were dissolved in $10 \mu \mathrm{L}$ DMSO to final concentration of $10 \mathrm{mg} / \mathrm{mL}$. $5 \mu \mathrm{l}$ (i.e. $40 \mathrm{nmol}$ ) dye in DMSO was added to the protein and incubated for $1 \mathrm{~h}$ at room temperature in a shaking block, covered with an aluminium foil to protect the dye from the light. Excess dye was removed by iterative buffer exchange using a 3K spin column (Amicon-Ultra-4 Centrifugal Filters Ultracell 3K, Millipore UFC800396) to PBS. The degree of labelling was inferred from measuring the OD.

\section{Cell preparation for immunofluorescence staining of $E$. coli cells with nanobodies}

For immunofluorescence staining, cells were subjected to a pH shift in order to induce Ldcl expression, as described in the "pH shift experiment" section above. 90 minutes after the $\mathrm{pH}$ shift, the OD600 of the cell culture was measured, and the volume of cells corresponding to $O D=4$, with $\mathrm{OD}=1$ corresponding to about $8 \times 10^{8}$ cells per $\mathrm{mL}$, were collected by centrifugation. After removal of LB by pipetting, cells were resuspended in $2 \mathrm{~mL}$ of $4 \%$ FA in PBS (made from 16\% Formaldehyde solution, Methanol-free from Thermo Scientific). Falcons were placed on a rotor for constant agitation for $45 \mathrm{~min}$ at room temperature. After fixation, cells were collected by 
centrifugation and the solution was removed by pipetting. Cells were then resuspended in $14 \mathrm{~mL}$ PBS (Gibco, Thermo Scientific) to remove and dilute the fixative. Cells were permeabilised for 10 min using $2 \mathrm{~mL} 0.1 \%$ Triton X-100 in PBS and subsequently washed three times with $10 \mathrm{~mL}$ PBS. Finally, cells were transferred to $1.5 \mathrm{~mL}$ tubes, centrifuged and resuspended in $200 \mu \mathrm{L} 1 \%$ BSA/PBS (BSA/PBS solution was dissolved for $30 \mathrm{~min}$ and sterile-filtered to avoid clumps). After $30 \mathrm{~min}$ of incubation, $0.5 \mu \mathrm{g}$ anti-Ldcl-Nb, labelled with the dedicated dye, was added to the $200 \mu \mathrm{L}$ bacteria-BSA/PBS suspension. Cells were incubated with the labelled anti-Ldcl-Nb for $16 \mathrm{~h}$ at $4^{\circ} \mathrm{C}$. The next day, cells were washed three times with $1 \mathrm{~mL}$ PBS, centrifuged to remove antibody solution and resuspended in $250 \mu \mathrm{l}$ PBS. When needed, Hoechst 33342 (Sigma-Aldrich) was added to a final concentration of about $100 \mathrm{ng} / \mathrm{mL}$.

\section{Wide-field imaging and flow cytometry}

For epifluorescence imaging, $2 \mu \mathrm{L}$ of cells were placed between a glass slide and a coverslip, which have been carefully pressed together, and observed using an inverted IX81 microscope, with a UPLFLN 100X oil immersion objective (N.A. 1.3) (Olympus), using the appropriate specific excitation and emission filters for AF488 (GFP-3035B set, Semrock) and DAPI (DAPI-5060B set, Semrock). Acquisitions were performed with Volocity software (QuorumTechnologies ${ }^{\mathrm{TM}}$ ) with a sCMOS 2048x2048 camera (Hamamatsu ORCA Flash 4, 16 bits/pixel) achieving a final magnification of $64 \mathrm{~nm}$ per pixel. For flow cytometry, $50 \mu \mathrm{L}$ of cells suspensions were injected in a MACSQuant VYB flow cytometer (Miltenyi Biotech, Bergish Gladbach, Germany) using the $488 \mathrm{~nm}$ excitation and 525 (50) nm emission channel (B1). AF488 positive populations were estimated after forward scatter (FSC) and side scatter (SSC) gating on the cells. Data were further processed with MACSQuantify software (Miltenyi Biotech).

\section{STORM imaging}

For Single Molecule Localization Microscopy, cells were transferred to a glucose buffer containing $50 \mathrm{mM} \mathrm{NaCl}, 150 \mathrm{mM}$ Tris (pH 8.0), 10\% Glucose, 100 mM MEA (Mercaptoethylamine) and 1x Glox. Glox was prepared as a $10 x$ stock and contained $1 \mu \mathrm{M}$ catalase and $2.3 \mu \mathrm{M}$ glucoseoxidase. Menzel glass slides (Thermo Scientific) and precision coverslips $(1.5 \mathrm{H}$ from ThorLabs) were cleaned for 30 min using an UV-ozone cleaning device (HELIOS-500, UVOTECH Systems). $2 \mu \mathrm{L}$ of immuno-labelled cells were placed onto a glass slide and covered with the coverslip, then cells were carefully spread by pressing glass slides firmly together and the sides were sealed with transparent nail polish to avoid evaporation. Mounted samples were imaged on a homemade SMLM set up based on an IX81 microscope (Olympus). STORM was performed by focusing a 643 $\mathrm{nm}$ excitation laser beam (Toptica Diode laser) to the back focal plane of an oil immersion UAPON100X (N.A. 1.49) objective. The intensity of the laser was tuned using an Acousto-Optical Tunable Filter (OATF, Quanta Tech). Acquisition was obtained with a 16 bits/pixel Evolve 512 EMCCD (Photometrics) using Metamorph (Molecular Devices), for a final pixel size of $123 \mathrm{~nm}$. 3DSTORM based on point-spread-function astigmatism (69) was performed using a cylindrical lens (LJ1516L1-A, Thorlabs) placed in the detection light path. STORM datasets consisting of about 30,000 frames, using a $643 \mathrm{~nm}$ laser power density of $3 \mathrm{~kW} / \mathrm{cm}^{2}$ and a $405 \mathrm{~nm}$ laser power density of up to $1 \mathrm{~W} / \mathrm{cm}^{2}$ and a frametime of $50 \mathrm{~ms}$, were acquired with an EMCCD gain set to 200. 3D 
point spread function calibration was achieved using tetraspec beads. Finally, data were processed with the Thunderstorm plugin (70) in Image J (71). 3D-images were rendered with Visp (72) using a minimum neighbour density threshold of 20 to 28 . A total of about 25,000 localisations with a median localisation precision of $\sim 17 \mathrm{~nm}$ were recorded per cell. A quantitative evaluation of molecular copy number from STORM data is notoriously difficult due to pronounced and potentially environmentally-dependent blinking of AF647. However, assuming an average of 3 blinking events per molecule and 100\% efficient labelling, one can estimate the number of Ldcl decamers in the range 500-1000 per cell, which would agree very well with earlier independent estimations $(6,31)$. Therefore, although the Ldcl labelling efficiency in cellulo was not precisely determined in this study, it is likely to be quite high, which is also consistent with our in vitro structural data on the Ldcl-nanobody complex.

\section{Biolayer interferometry measurements}

For BLI binding studies, RavA with a biotinylated C-terminal AviTag was expressed and purified as previously described (30). BLI experiments were performed in 1x HBS pH at 7.0 (25 mM HEPES, $300 \mathrm{mM} \mathrm{NaCl}, 10 \mathrm{mM} \mathrm{MgCl}$, $10 \%$ glycerol) supplemented with 1x kinetics buffer (0.1\% w/v BSA, 0.02\% v/v Tween-20), 1mM ADP, 1mM DTT and 0.1 mM PLP. Experiments were performed using the BLItz System instrument (FortéBio), operated at room temperature. Before the start of each BLI experiment, RavA-AviTag was incubated with $1 \mathrm{mM}$ ADP for $10 \mathrm{~min}$. Streptavidin-coated biosensors (FortéBio) were functionalised with biotinylated RavA-AviTag, then quenched with 10 $\mu \mathrm{g} / \mathrm{mL}$ biocytin. Experiments with the wild-type Ldcl are from (30). For C-terminal Ldcl-FP fusions, pins were dipped in wells containing a range of Ldcl-FP concentrations from 0 to $1000 \mathrm{nM}$, with no binding signal recorded at any concentration of Ldcl-FP.

\section{Data and code availability}

Cryo-EM maps, along with the corresponding fitted atomic structures, have been submitted to the EMDB and PDB with accession codes EMD-10850 and PDB-6YN6 for Ldcl stacks (pH 5.7), and EMD-10849 and PDB-6YN5 for Ldcl decamers (pH 7.0).

\section{Acknowledgements}

We thank Guy Schoehn for establishing and managing the cryo-electron microscopy platform and for providing training and support, and Rose-Laure Revel-Goyet and Françoise Lacroix for the support and access to the M4D Cell imaging Platform. We are grateful to Aymeric Peuch for help with the usage of the EM computing cluster and Daniel Thédie, Kévin Floc'h and Joel Beaudoin for discussions. We thank Alain Roussel and Aline Desmyter for the nanobody production, and Julien Perard for help with the cloning of the Dendra2 ${ }_{\text {T69A }}$-Ldcl construct. We acknowledge the European Synchrotron Radiation Facility for provision of beam time on CM01. This work was funded by the European Union's Horizon 2020 research and innovation programme under grant agreement No 647784 to IG. The nanobody generation platform of the AFMB laboratory (Marseille, France) was supported by the French Infrastructure for Integrated Structural Biology (FRISBI) ANR-10-INSB-05-01. For electron and fluorescence microscopy studies, this work used the platforms of the Grenoble Instruct centre (ISBG; UMS 3518 CNRS-CEA-UJF-EMBL) 
with support from FRISBI (ANR-10-INSB-05-02) and GRAL (ANR-10-LABX-49-01) within the Grenoble Partnership for Structural Biology (PSB). The electron microscope facility (Glacios electron microscope) is supported by the Rhône-Alpes Region (CIBLE and FEDER), the FRM, the CNRS, the University of Grenoble and the GIS-IBISA. JF was supported by a long-term EMBO fellowship (ALTF441-2017) and a Marie Skłodowska-Curie actions Individual Fellowship (789385, RespViRALI).

\section{Author Contributions}

C.L., M.B., V.A., A.F. and K.H cloned constructs, M.J., A.F. and K.H. purified proteins. M.J. and I.G. performed ns-EM imaging and analysis. A.D., G.E. and M.B.-V. performed cryo-EM imaging. J.F. and A.D. performed cryo-EM analysis. M.J. and J.F. built models resulting from EM maps. M.J., J.F. and I.G. analysed structures and interpreted data. C.L. and M.B. performed optical imaging of overexpression constructs with input of V.A., J.-P.K. and D.B. C.L. performed nanobody characterisation for optical imaging with input from J.-P.K. C.L. analysed endogenous expression and performed optical imaging of endogenous Ldcl with input of V.A., J.-P.K. and D.B. C.L. and D.B. analysed STORM Images. M.J., C.L., J.F. and I.G. analysed the data and prepared the figures and tables. C.L. and D.B. contributed to the design of the optical imaging part of the project together with I.G. I.G. designed, supervised and funded the overall study. M.J., J.F. and I.G. wrote the manuscript with significant input from C.L. and contributions from all of the authors.

\section{References}

1. M. Prouteau, R. Loewith, Regulation of Cellular Metabolism through Phase Separation of Enzymes. Biomolecules 8, 160 (2018).

2. F. C. Fang, E. R. Frawley, T. Tapscott, A. Vázquez-Torres, Bacterial Stress Responses during Host Infection. Cell Host Microbe 20, 133-143 (2016).

3. N. Watson, D. S. Dunyak, E. L. Rosey, J. L. Slonczewski, E. R. Olson, Identification of elements involved in transcriptional regulation of the Escherichia coli cad operon by external pH. J. Bacteriol. 174, 530-540 (1992).

4. J. W. Foster, Escherichia coli acid resistance: Tales of an amateur acidophile. Nat. Rev. Microbiol. 2, 898-907 (2004).

5. P. L. Moreau, The lysine decarboxylase CadA protects Escherichia coli starved of phosphate against fermentation acids. J. Bacteriol. 189, 2249-2261 (2007).

6. U. Kanjee, et al., Linkage between the bacterial acid stress and stringent responses: The structure of the inducible lysine decarboxylase. EMBO J. 30, 931-944 (2011).

7. E. F. Gale, The Bacterial Amino Acid Decarboxylases. Adv. Enzymol. - Relat. Areas Mol. Biol. 6, 1-32 (1946).

8. D. L. Sabo, E. A. Boeker, B. Byers, H. Waron, E. H. Fischer, Purification and Physical Properties of Inducible Escherichia coli Lysine Decarboxylase. Biochemistry 13, 662-670 (1974).

9. E. F. Gale, H. M. R. Epps, The effect of the $\mathrm{pH}$ of the medium during growth on the enzymic activities of bacteria (Escherichia coli and Micrococcus lysodeikticus) and the biological significance of the changes produced. Biochem. J. 36, 600-618 (1942).

10. E. Kandiah, et al., Structural insights into the Escherichia coli lysine decarboxylases and molecular 
determinants of interaction with the AAA+ATPase RavA. Sci. Rep. 6 (2016).

11. B. Zhao, Investigation of the Regulation of the Lysine Decarboxylase Ldcl Activity by the Alarmone ppGpp and MoxR Family AAA + ATPase RavA (2012).

12. I. V. Surovtsev, C. Jacobs-Wagner, Subcellular Organization: A Critical Feature of Bacterial Cell Replication. Cell 172, 1271-1293 (2018).

13. W. Margolin, The price of tags in protein localization studies. J. Bacteriol. 194, 6369-6371 (2012).

14. A. Gahlmann, W. E. Moerner, Exploring bacterial cell biology with single-molecule tracking and superresolution imaging. Nat. Rev. Microbiol. 12, 9-22 (2014).

15. D. Landgraf, B. Okumus, P. Chien, T. A. Baker, J. Paulsson, Segregation of molecules at cell division reveals native protein localization. Nat. Methods 9, 480-482 (2012).

16. L. M. Costantini, M. Fossati, M. Francolini, E. L. Snapp, Assessing the Tendency of Fluorescent Proteins to Oligomerize Under Physiologic Conditions. Traffic 13, 643-649 (2012).

17. M. Ingerson-Mahar, A. Briegel, J. N. Werner, G. J. Jensen, Z. Gitai, The metabolic enzyme CTP synthase forms cytoskeletal filaments. Nat. Cell Biol. 12, 739-746 (2010).

18. E. M. Lynch, et al., Human CTP synthase filament structure reveals the active enzyme conformation. Nat. Struct. Mol. Biol. 24, 507-514 (2017).

19. T. G. Frey, D. Eisenberg, F. A. Eiserling, Glutamine synthetase forms three and seven stranded helical cables. Proc. Natl. Acad. Sci. U. S. A. 72, 3402-3406 (1975).

20. G. Kim, et al., Aldehyde-alcohol dehydrogenase forms a high-order spirosome architecture critical for its activity. Nat. Commun. 10 (2019).

21. K. Schuchmann, J. Vonck, V. Müller, A bacterial hydrogen-dependent CO2 reductase forms filamentous structures. FEBS J. 283, 1311-1322 (2016).

22. Z. Yao, R. Carballido-López, Fluorescence Imaging for Bacterial Cell Biology: From Localization to Dynamics, From Ensembles to Single Molecules. Annu. Rev. Microbiol. 68 (2014).

23. M. J. Rust, M. Bates, X. Zhuang, Sub-diffraction-limit imaging by stochastic optical reconstruction microscopy (STORM). Nat. Methods 3, 793-795 (2006).

24. E. Betzig, et al., Imaging intracellular fluorescent proteins at nanometer resolution. Science $\mathbf{3 1 3}$, 1642-5 (2006).

25. S. T. Hess, T. P. K. Girirajan, M. D. Mason, Ultra-high resolution imaging by fluorescence photoactivation localization microscopy. Biophys. J. 91, 4258-4272 (2006).

26. H. Chang, et al., A unique series of reversibly switchable fluorescent proteins with beneficial properties for various applications. Proc. Natl. Acad. Sci. 109, 4455-4460 (2012).

27. R. Berardozzi, V. Adam, A. Martins, D. Bourgeois, Arginine 66 Controls Dark-State Formation in Green-to-Red Photoconvertible Fluorescent Proteins. J. Am. Chem. Soc. 138, 558-565 (2016).

28. H. Malet, et al., Assembly principles of a unique cage formed by hexameric and decameric E. coli proteins. Elife 3, e03653 (2014).

29. J. Snider, et al., Formation of a distinctive complex between the inducible bacterial lysine decarboxylase and a novel AAA+ ATPase. J. Biol. Chem. 281, 1532-1546 (2006).

30. M. Jessop, et al., Structural insights into ATP hydrolysis by the MoxR ATPase RavA and the LdclRavA cage-like complex. Commun. Biol. 3, 46 (2020).

31. M. El Bakkouri, et al., Structure of RavA MoxR AAA+ protein reveals the design principles of a molecular cage modulating the inducible lysine decarboxylase activity. Proc. Natl. Acad. Sci. 107, 
22499-22504 (2010).

32. K. S. Wong, et al., The MoxR ATPase RavA and its cofactor ViaA interact with the NADH: Ubiquinone oxidoreductase I in Escherichia coli. PLoS One 9, e85529 (2014).

33. K. S. Wong, V. Bhandari, S. C. Janga, W. A. Houry, The RavA-ViaA Chaperone-Like System Interacts with and Modulates the Activity of the Fumarate Reductase Respiratory Complex. J. Mol. Biol. 429, 324-344 (2017).

34. M. Zhang, et al., Rational design of true monomeric and bright photoactivatable fluorescent proteins. Nat. Methods 9 (2012).

35. C. Coltharp, J. Xiao, Superresolution microscopy for microbiology. Cell. Microbiol. 14, 1808-1818 (2012).

36. K. Cramer, et al., Visualization of Bacterial Protein Complexes Labeled with Fluorescent Proteins and Nanobody Binders for STED Microscopy. Int. J. Mol. Sci. 20, 3376 (2019).

37. U. Kanjee, I. Gutsche, S. Ramachandran, W. A. Houry, The enzymatic activities of the escherichia coli basic aliphatic amino acid decarboxylases exhibit a pH zone of inhibition. Biochemistry 50, 93889398 (2011).

38. I. Barák, K. Muchová, The role of lipid domains in bacterial cell processes. Int. J. Mol. Sci. 14, 40504065 (2013).

39. T. Lenn, M. C. Leake, C. W. Mullineaux, Clustering and dynamics of cytochrome bd-I complexes in the Escherichia coli plasma membrane in vivo. Mol. Microbiol. 70, 1397-1407 (2008).

40. H. Erhardt, et al., Organization of the Escherichia coli aerobic enzyme complexes of oxidative phosphorylation in dynamic domains within the cytoplasmic membrane. Microbiologyopen 3, 316-326 (2014).

41. I. Llorente-Garcia, et al., Single-molecule in vivo imaging of bacterial respiratory complexes indicates delocalized oxidative phosphorylation. Biochim. Biophys. Acta - Bioenerg. 1837, 811-824 (2014).

42. J. N. Werner, et al., Quantitative genome-scale analysis of protein localization in an asymmetric bacterium. Proc. Natl. Acad. Sci. 106, 7858-7863 (2009).

43. A. Magalon, F. Alberge, Distribution and dynamics of OXPHOS complexes in the bacterial cytoplasmic membrane. Biochim. Biophys. Acta - Bioenerg. 1857, 198-213 (2016).

44. M. Bramkamp, D. Lopez, Exploring the Existence of Lipid Rafts in Bacteria. Microbiol. Mol. Biol. Rev. 79, 81-100 (2015).

45. H. Erhardt, et al., Disruption of individual nuo-genes leads to the formation of partially assembled NADH:ubiquinone oxidoreductase (complex I) in Escherichia coli. Biochim. Biophys. Acta 1817, 86371 (2012).

46. Y. Yamamoto, Y. Miwa, K. Miyoshi, J. I. Furuyama, H. Ohmori, The Escherichia coli IdcC gene encodes another lysine decarboxylase, probably a constitutive enzyme. Genes Genet. Syst. 72, 167172 (1997).

47. M. T. Swulius, G. J. Jensen, The helical mreb cytoskeleton in Escherichia coli MC1000/pLE7 is an artifact of the N-terminal yellow fluorescent protein tag. J. Bacteriol. 194, 6382-6386 (2012).

48. I. Barák, K. Muchová, A. J. Wilkinson, P. J. O’Toole, N. Pavlendová, Lipid spirals in Bacillus subtilis and their role in cell division. Mol. Microbiol. 68, 1315-1327 (2008).

49. E. Mileykovskaya, W. Dowhan, Cardiolipin membrane domains in prokaryotes and eukaryotes. Biochim. Biophys. Acta - Biomembr. 1788, 2084-2091 (2009). 
50. I. Fishov, C. L. Woldringh, Visualization of membrane domains in Escherichia coli. Mol. Microbiol. 32, 1166-1172 (1999).

51. R. A. Daniel, J. Errington, Control of cell morphogenesis in bacteria: Two distinct ways to make a rodshaped cell. Cell 113, 767-776 (2003).

52. S. Wang, L. Furchtgott, K. C. Huang, J. W. Shaevitz, Helical insertion of peptidoglycan produces chiral ordering of the bacterial cell wall. Proc. Natl. Acad. Sci. U. S. A. 109 (2012).

53. E. Pardon, et al., A general protocol for the generation of Nanobodies for structural biology. Nat. Protoc. 9, 674-693 (2014).

54. A. Desmyter, et al., Viral infection modulation and neutralization by camelid nanobodies. Proc. Natl. Acad. Sci. U. S. A. (2013) https:/doi.org/10.1073/pnas.1301336110.

55. J. A. Mindell, N. Grigorieff, Accurate determination of local defocus and specimen tilt in electron microscopy. J. Struct. Biol. 142, 334-347 (2003).

56. S. J. Ludtke, P. R. Baldwin, W. Chiu, EMAN: Semiautomated Software for High-Resolution SingleParticle Reconstructions. J. Struct. Biol. 128, 82-97 (1999).

57. S. H. W. Scheres, RELION: Implementation of a Bayesian approach to cryo-EM structure determination. J. Struct. Biol. 180, 519-530 (2012).

58. S. H. W. Scheres, "Processing of Structurally Heterogeneous Cryo-EM Data in RELION" in Methods in Enzymology, (2016), pp. 125-157.

59. E. F. Pettersen, et al., UCSF Chimera--a visualization system for exploratory research and analysis. J. Comput. Chem. 25, 1605-12 (2004).

60. E. Kandiah, et al., CM01: A facility for cryo-electron microscopy at the European synchrotron. Acta Crystallogr. Sect. D Struct. Biol. 75 (2019).

61. S. Q. Zheng, et al., MotionCor2: Anisotropic correction of beam-induced motion for improved cryoelectron microscopy. Nat. Methods 14, 331-332 (2017).

62. A. Rohou, N. Grigorieff, CTFFIND4: Fast and accurate defocus estimation from electron micrographs. J. Struct. Biol. 192, 216-221 (2015).

63. G. Tang, et al., EMAN2: An extensible image processing suite for electron microscopy. J. Struct. Biol. 157, 38-46 (2007).

64. J. Zivanov, et al., New tools for automated high-resolution cryo-EM structure determination in RELION-3. Elife 7, 1-22 (2018).

65. K. Zhang, Gctf: Real-time CTF determination and correction. J. Struct. Biol. 193, 1-12 (2016).

66. A. Punjani, J. L. Rubinstein, D. J. Fleet, M. A. Brubaker, CryoSPARC: Algorithms for rapid unsupervised cryo-EM structure determination. Nat. Methods 14, 290-296 (2017).

67. P. B. Rosenthal, R. Henderson, Optimal determination of particle orientation, absolute hand, and contrast loss in single-particle electron cryomicroscopy. J. Mol. Biol. 333, 721-745 (2003).

68. P. D. Adams, et al., PHENIX: A comprehensive Python-based system for macromolecular structure solution. Acta Crystallogr. Sect. D Biol. Crystallogr. 66, 213-221 (2010).

69. B. Huang, W. Wang, M. Bates, X. Zhuang, Three-dimensional super-resolution imaging by stochastic optical reconstruction microscopy. Science (80-. ). 319 (2008).

70. M. Ovesný, P. Křížek, J. Borkovec, Z. Švindrych, G. M. Hagen, ThunderSTORM: A comprehensive ImageJ plug-in for PALM and STORM data analysis and super-resolution imaging. Bioinformatics $\mathbf{3 0}$ (2014). 

made available under aCC-BY-ND 4.0 International license.

840 71. C. A. Schneider, W. S. Rasband, K. W. Eliceiri, NIH Image to ImageJ: 25 years of image analysis. Nat. Methods 9 (2012).

72. M. El Beheiry, M. Dahan, ViSP: Representing single-particle localizations in three dimensions. Nat. Methods 10 (2013). 
bioRxiv preprint doi: https://doi.org/10.1101/2020.05.12.090381; this version posted July 9, 2020. The copyright holder for this preprint (which was not certified by peer review) is the author/funder, who has granted bioRxiv a license to display the preprint in perpetuity. It is made available under aCC-BY-ND 4.0 International license.

Figure 1.

A Dendra2 $2_{\text {T69A }}$-LdcI
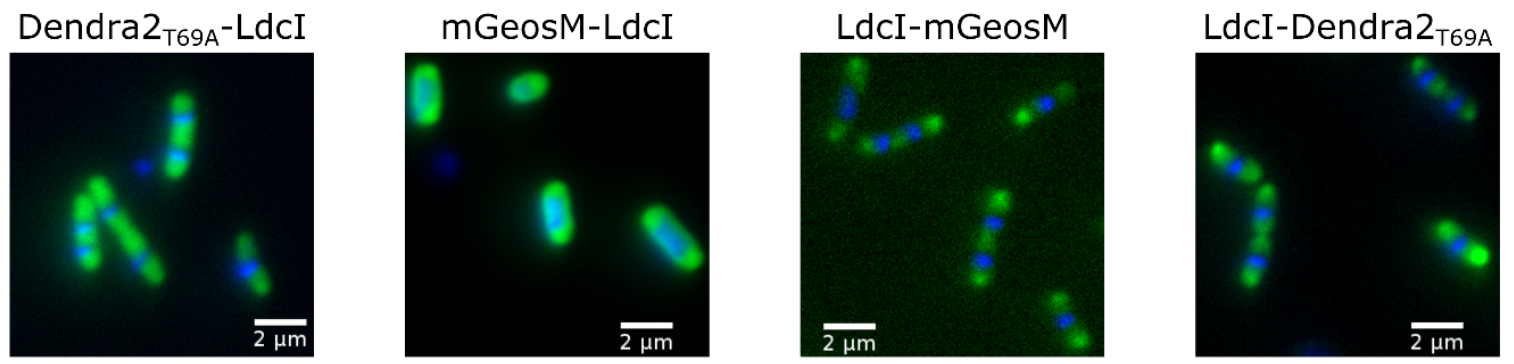

B
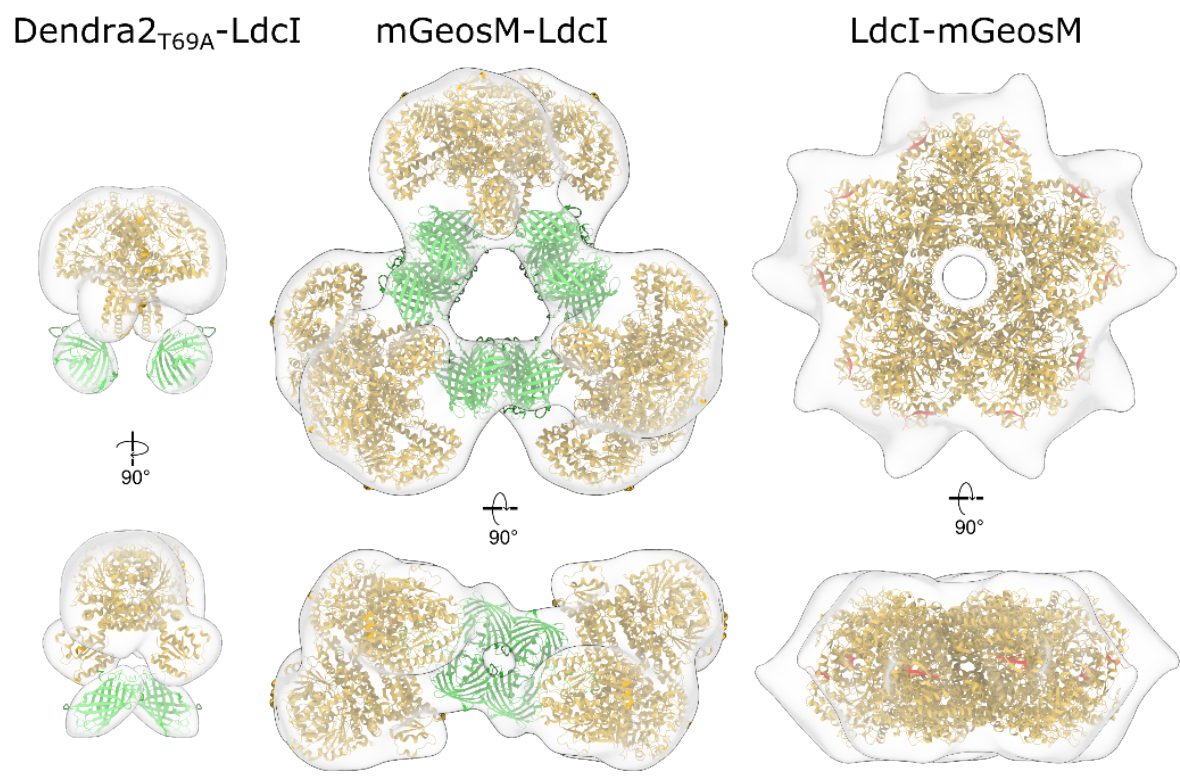

LdcI-Dendra2 $2_{\text {T69A }}$

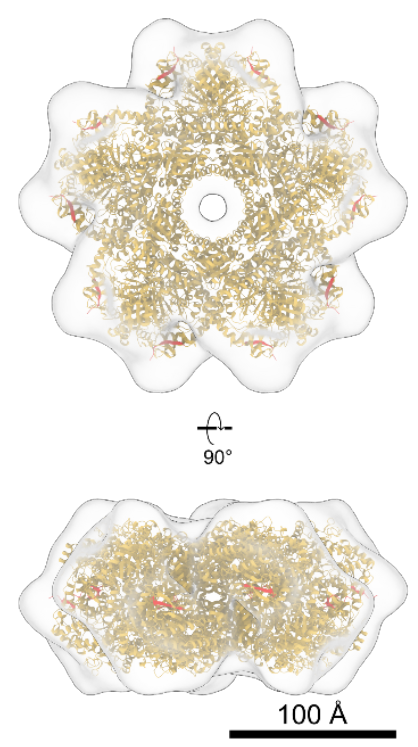

Figure 1. Fluorescent protein fusions affect Ldcl structure without significantly altering cellular localisation of overexpressed constructs. A) Wide-field fluorescence microscopy images of $E$. coli cells overexpressing the fluorescent fusion proteins in A). Green fluorescence = FP-Ldcl, blue fluorescence = DAPI-stained DNA. B) ns-EM 3D maps of fluorescently-tagged Ldcl, with fitted models. Left - Dendra2 ${ }_{\text {T69A }}-\mathrm{Ldcl}$ forms dimers, with fluorescent barrels located next to the N-terminus of Ldcl as expected. Second from left - mGeos-M-Ldcl forms large non-native oligomers, composed of three Ldcl tetramers bridged by tetramers of mGeosM. Second from right and right - both C-terminal fluorescent fusions Ldcl-mGeosM and Ldcl-Dendra $2_{\text {T69A }}$ form decamers, with protrusions at the C-terminus (coloured in red) attributed to flexibly-linked fluorescent proteins. Fitted PBD models are as follows - Ldcl: 3N75; Dendra2: 2VZX; mGeosM: $3 \mathrm{~S} 05$ (mEos2 crystal structure). 
Figure 2.

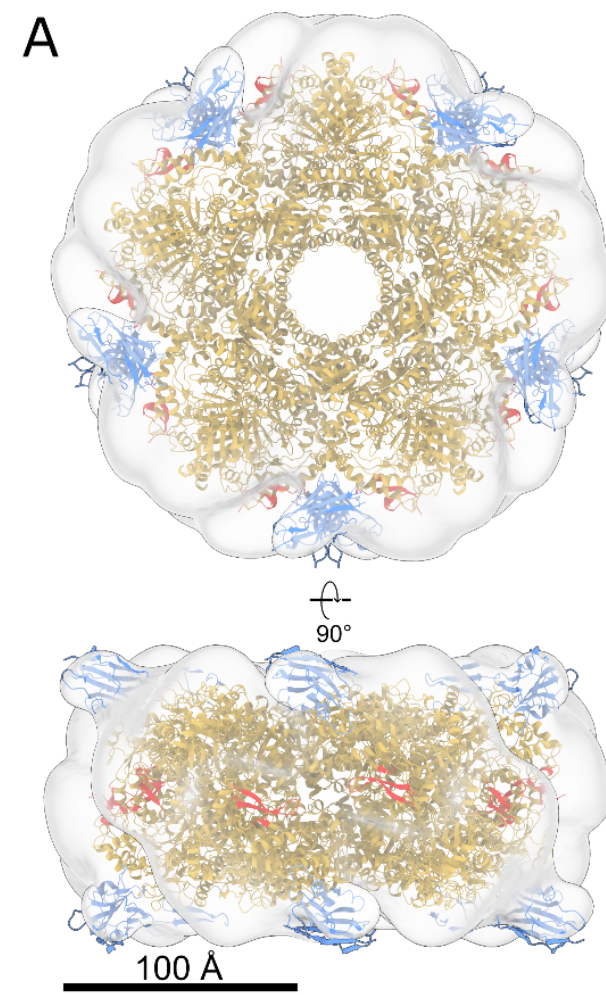

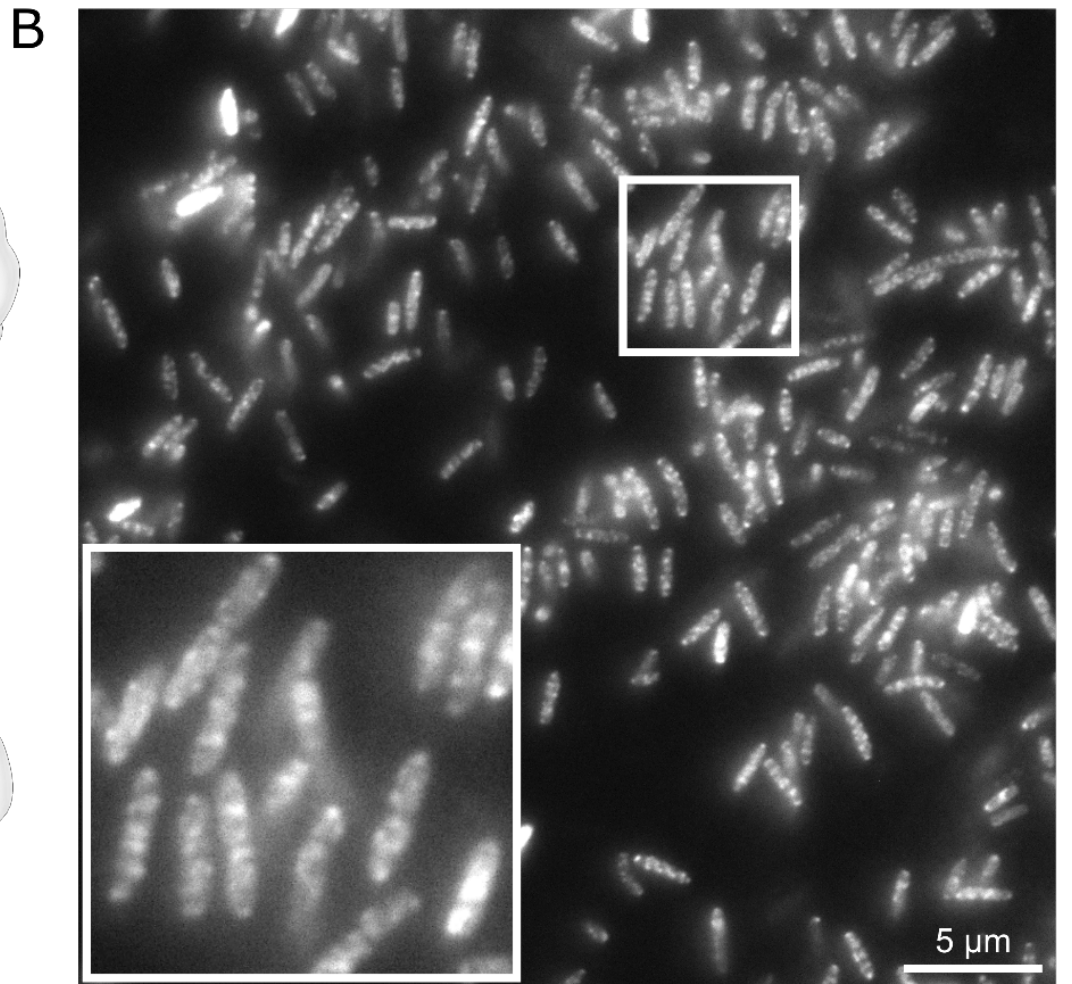

Figure 2. Anti-Ldcl-Nb is a useful tool to probe cellular localisation of endogenous Ldcl under acid stress conditions. A) Ns-EM 3D reconstruction of the Ldcl decorated by anti-LdclNb. An Ldcl decamer (gold, PDB ID: 3N75) and 10 nanobodies (blue, PDB ID: 1MEL, antilysozyme nanobody) are fitted in the density, with the nanobody binding at the top and bottom of the decameric ring. The C-terminal RavA binding site is indicated in red, and is in a spatially distinct location from the bound nanobodies. Scale bar $=100 \AA$. B) Wide-field fluorescence microscopy image of wild-type E. coli MG1655 cells grown at $\mathrm{pH} 4.6$ for 90 minutes stained with anti-Ldcl-Nb labelled with AF488. Inset - zoom of the image showing punctuate fluorescence patterns. Scale bar $=5 \mu \mathrm{m}$. 
Figure 3.

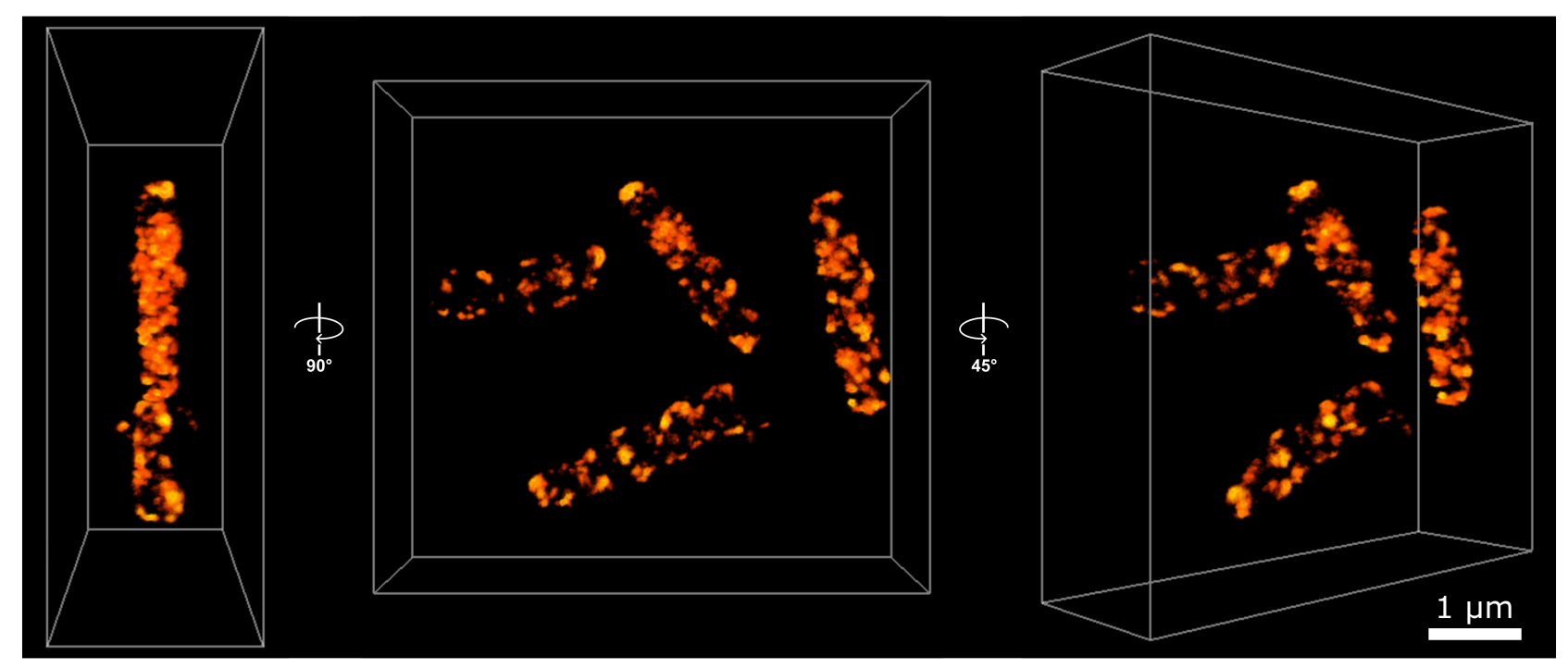

Figure 3. 3D STORM imaging of $E$. coli cells stained with anti-Ldcl-Nb reveal a patchy pseudo-helically arranged distribution of endogenous Ldcl upon acid stress. 3D-STORM imaging of native Ldcl in wild-type E. coli cells 90 minutes after exposure to acid stress (see Methods) with AF647-conjugated anti-Ldcl-Nb. Points are coloured according to localisation density, with brighter points corresponding to higher localisation density. The centre panel shows four cells in the field of view, looking down the z-axis. Left and right panels show side and tilted views respectively. Scale bar $=1 \mathrm{~nm}$. 
Figure 4.
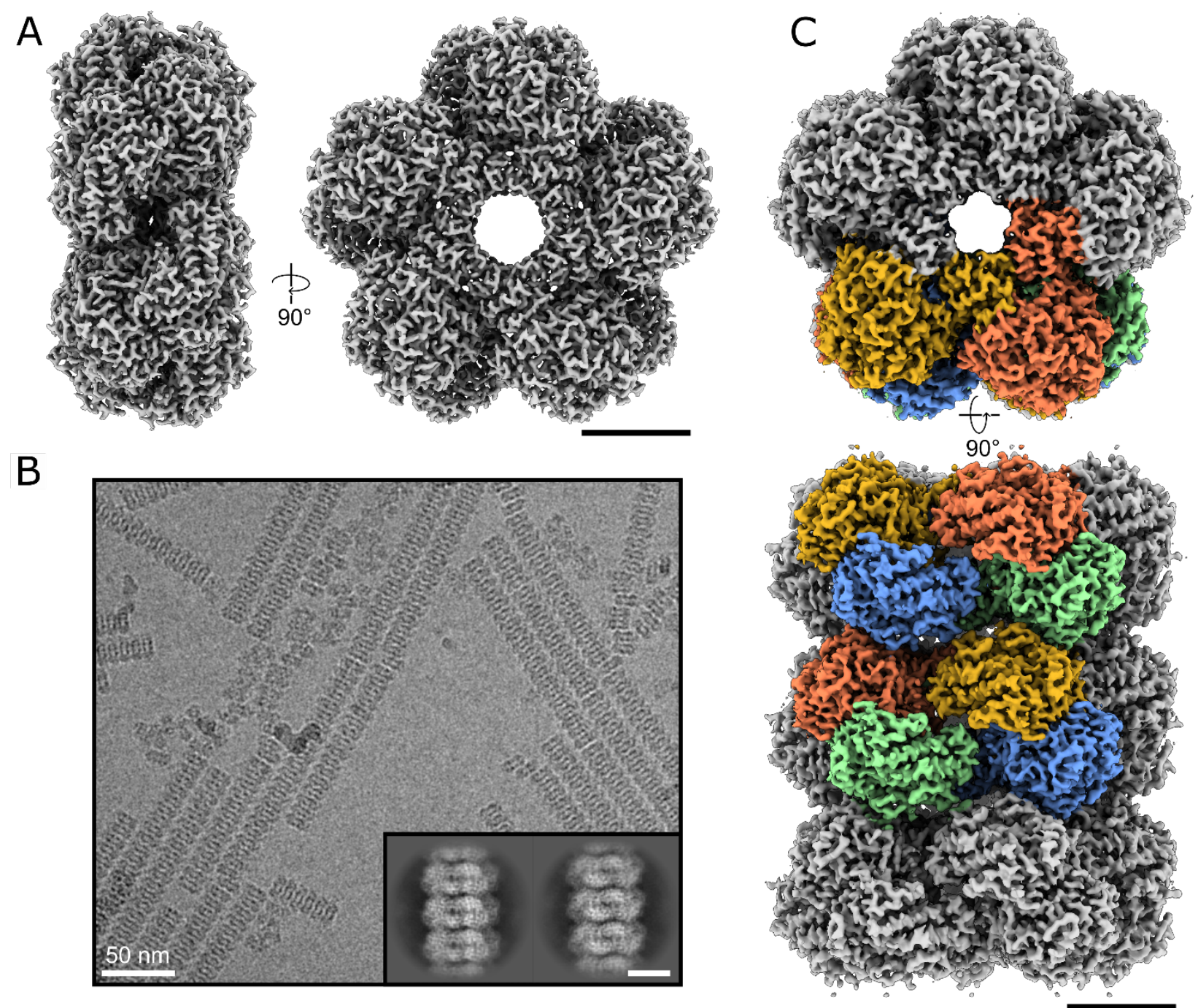

D
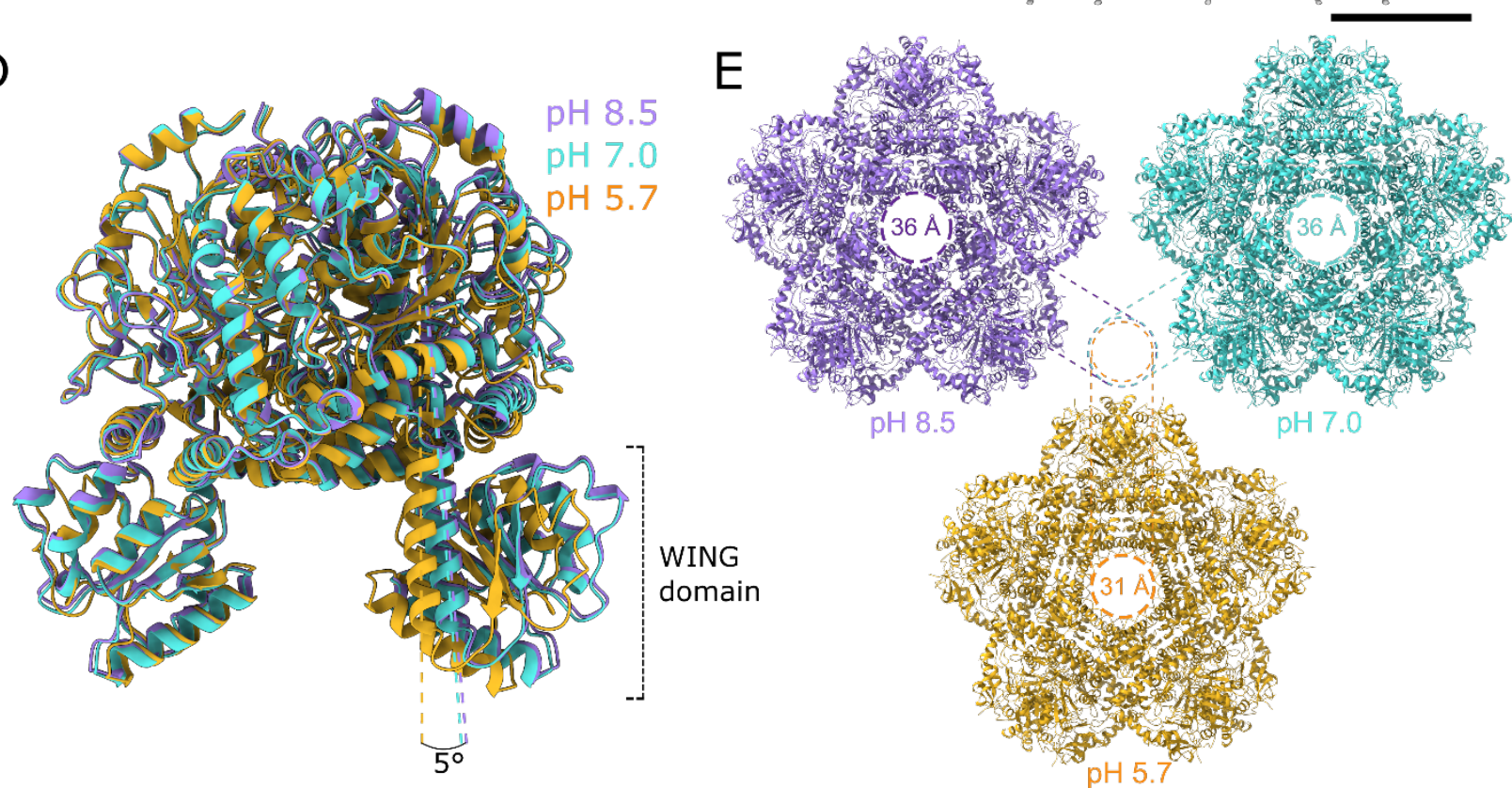

Figure 4. Cryo-EM analysis of the Ldcl decamer at neutral $\mathrm{pH}$ and Ldcl stacks formed in acid stress conditions. A) Cryo-EM reconstruction of the Ldcl decamer at $\mathrm{pH} 7.0$ from side (left) and top (right) views. Scale bar $=50 \AA$. B) Cryo-EM micrograph of Ldcl stacks at pH 5.7, scale bar $=$ $100 \mathrm{~nm}$. Inset - 2D class averages displaying clear secondary structural features, scale bar $=100$ A. C) Top (above) and side (below) views of the cryo-EM reconstruction of a three-decamer Ldcl 
stack. Four dimers are coloured either blue/gold or green/coral, corresponding to the colouring of the atomic model presented in Figure 5. Scale bar $=50 \AA$. D) Overlay of Ldcl dimers at pH 8.5 (PDB ID: 3N75, shown in lilac), 7.0 (shown in cyan) and 5.7 (shown in gold). Alignment was carried out on a single monomer in the dimer pair. There is a $5^{\circ}$ shift in the angle between the wing domains of $\mathrm{Ldcl}$ at $\mathrm{pH} 5.7$ and $\mathrm{pH} 7.0 / 8.5$. E) Comparison of the central decamer pore diameter between $\mathrm{Ldcl}$ at $\mathrm{pH} 8.5,7.0$ and 5.7, showing a $5 \AA$ decrease in the pore size upon stack formation at low $\mathrm{pH}$. 
Figure 5.
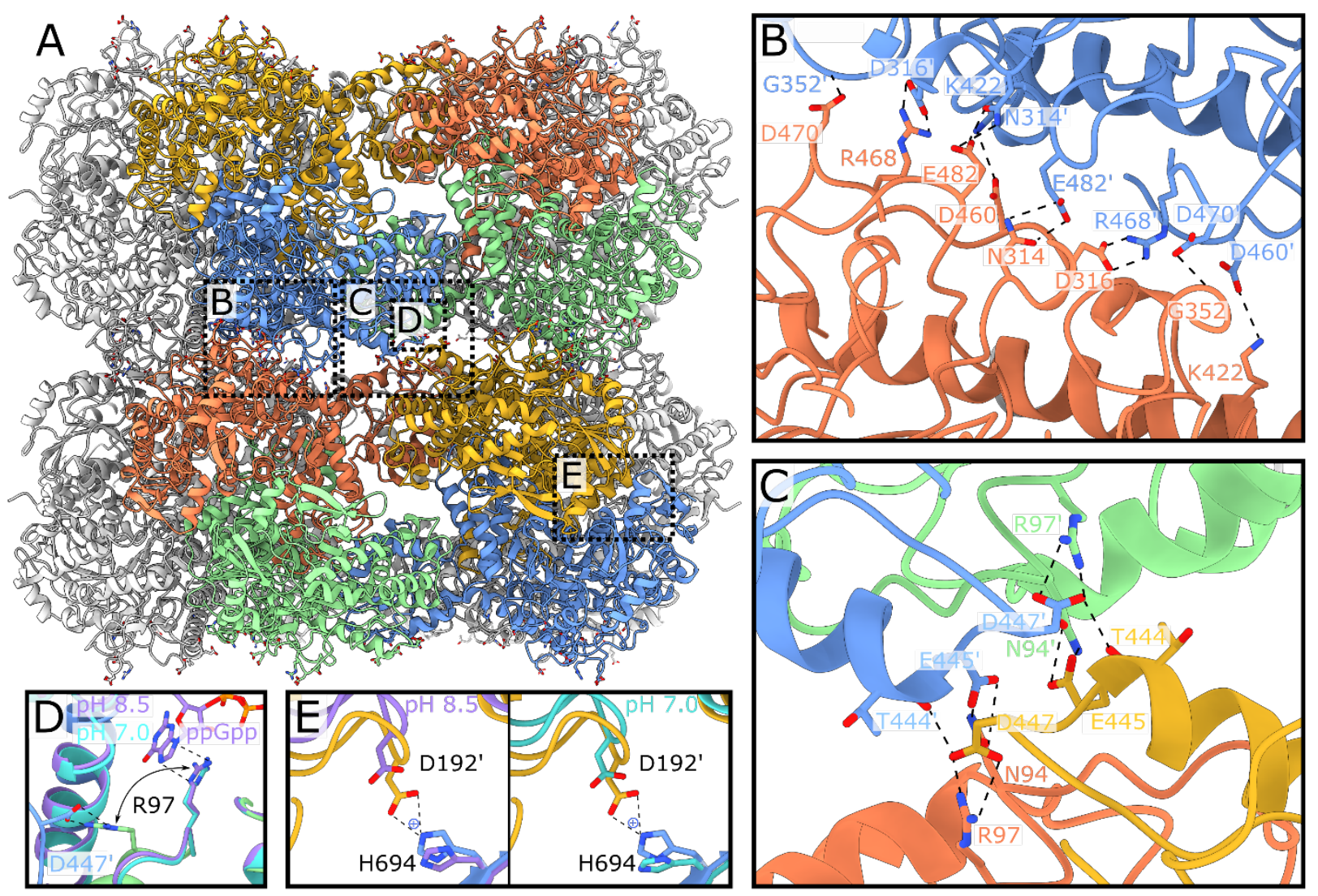

Figure 5. Structural insights into molecular determinants of the Ldcl polymerisation under acid stress conditions. A) Atomic model of a two-decamer Ldcl stack at pH 5.7. Dimers are coloured as shown in the cryo-EM map in Figure 4. Dotted boxes on the two-decamer stack indicate the locations of the zooms shown in panels B-E. B) Close-up of the first decamer-decamer interface, which includes the key stack-forming residue R468. C) Close-up of the second decamerdecamer interface. D) Overlay of the Ldcl decamer structures at pH 8.5 and 7.0 with the Ldcl stack structure at $\mathrm{pH}$ 5.7, focussed on R97. R97 in the Ldcl stack (green) adopts a different conformation compared to the one in the $\mathrm{pH} 8.5$ crystal structure (purple, with ppGpp bound) and the $\mathrm{pH} 7.0$ cryo-EM map (cyan, without ppGpp bound). Despite the absence of ppGpp in the $\mathrm{pH} 7.0$ sample, R97 is still oriented towards the ppGpp binding site. E) Comparison between the H694-D192' distance in the Ldcl stack at pH 5.7 (coloured gold and blue), the Ldcl decamer at pH 8.5 (left, coloured purple) and $\mathrm{pH} 7.0$ (right, coloured cyan). Key residues are labelled for all panels, and interactions are shown with dotted lines. 
Figure 6.
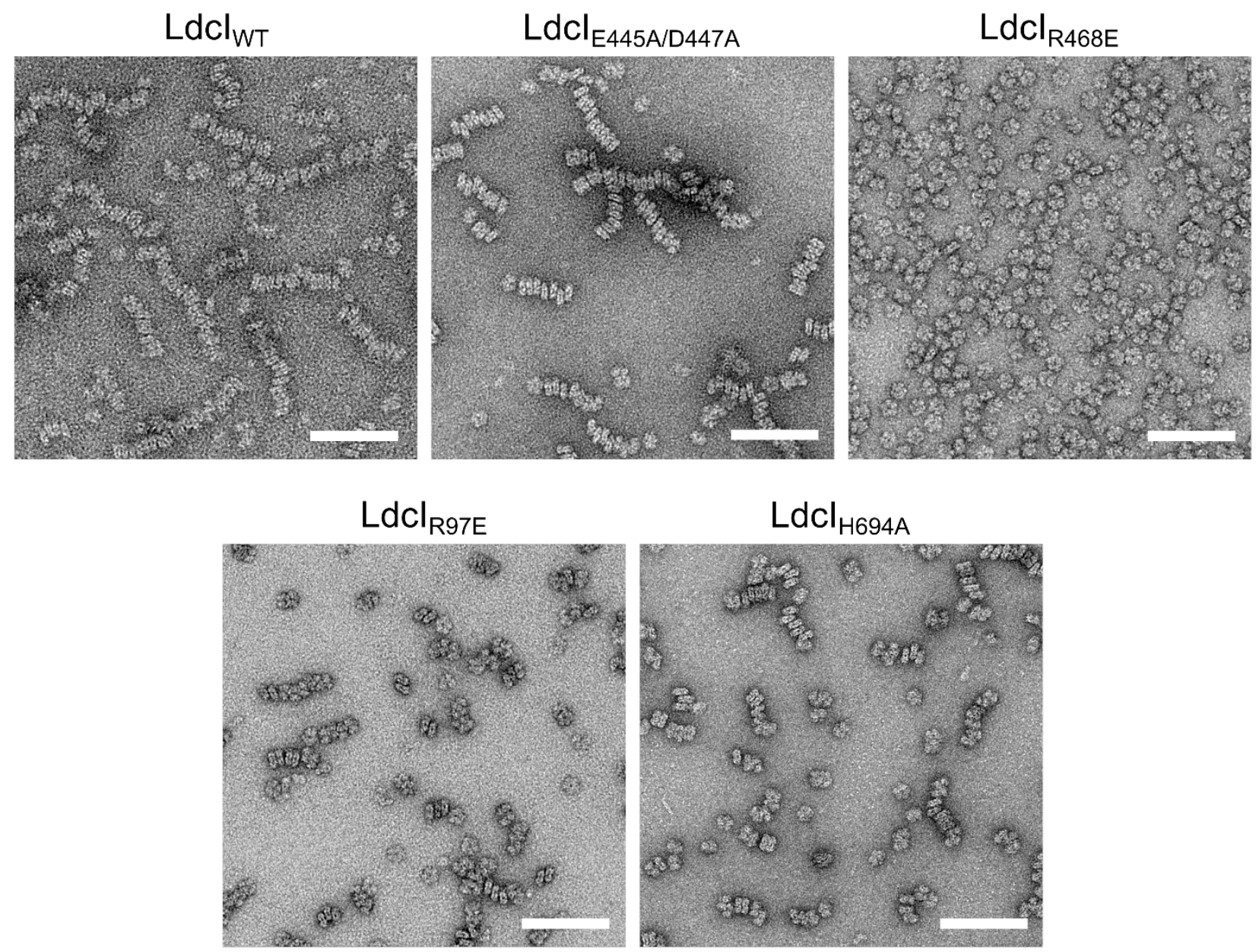

Figure 6. Mutational analysis of the predicted molecular determinants of the Ldcl polymerisation under acid stress conditions Cropped negative stain EM micrographs of wildtype and mutant $\mathrm{Ldcl}$ at $\mathrm{pH} 5.7$, scale bar $=100 \mathrm{~nm}$. Ldcl $\mathrm{wT}_{\mathrm{T}}$ polymerises at $\mathrm{pH} 5.7$, as does the

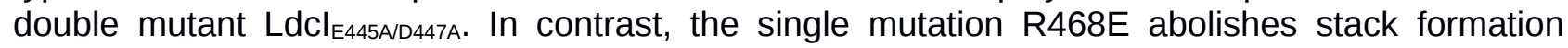
completely. Both $\mathrm{Ldcl}_{\mathrm{R} 9 \mathrm{E}}$ and $\mathrm{LdCl}_{\mathrm{H} 964 \mathrm{~A}}$ are able to polymerise, however the stacks tend to be shorter than for $\mathrm{Ldcl}_{\text {WT. }}$ Micrographs for $\mathrm{Ldcl}_{\mathrm{R} 97 \mathrm{E} / \mathrm{R} 468 \mathrm{E}}$, Ldcl $\mathrm{Ld45A/D447A/R468E}_{\text {, }}$ and $\mathrm{Ldcl}_{\mathrm{H} 694 \mathrm{~N}}$ were also collected but are not shown here. Ldcl E445AID447A/R468E $_{\text {and }}$ Ldcl $_{\text {R97E/R468E }}$ behaved like Ldcl R468E $_{\text {and }}$ remained entirely decameric at low $\mathrm{pH}$, while $\mathrm{Ldcl}_{\mathrm{H} 694 \mathrm{~N}}$ displayed similar behaviour to $\mathrm{Ldcl}_{\mathrm{H} 694 \mathrm{~A}}$. 


\section{Supplementary Figure 1.}

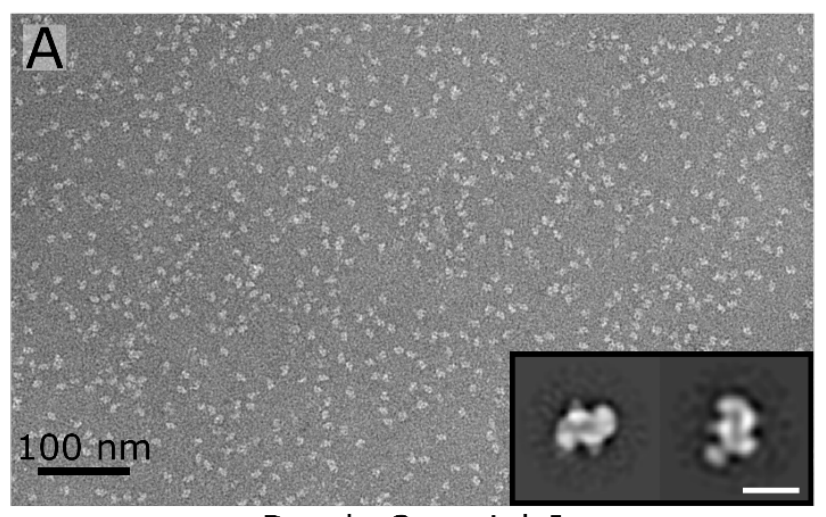

Dendra $2_{T 69 A}$-LdcI

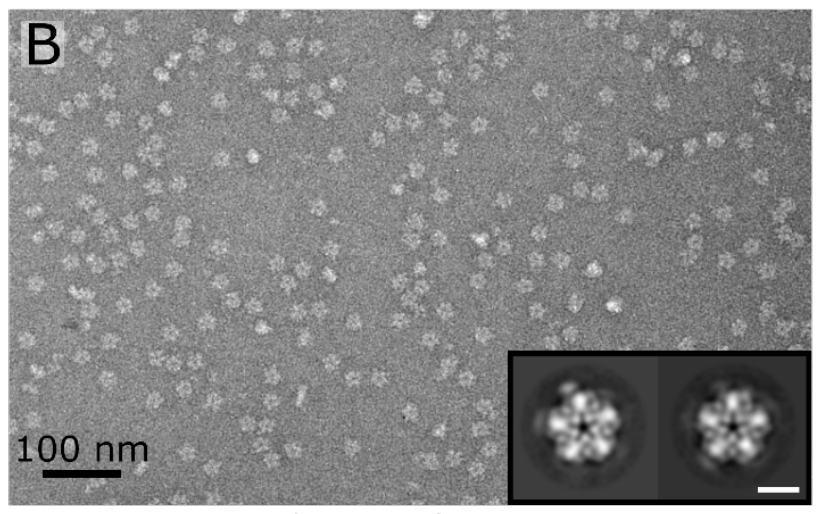

LdcI-Dendra2 $2_{\mathrm{T} 69 \mathrm{~A}}$

C

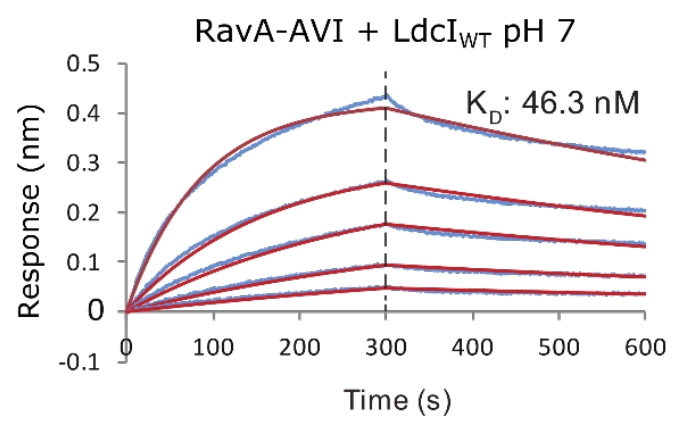

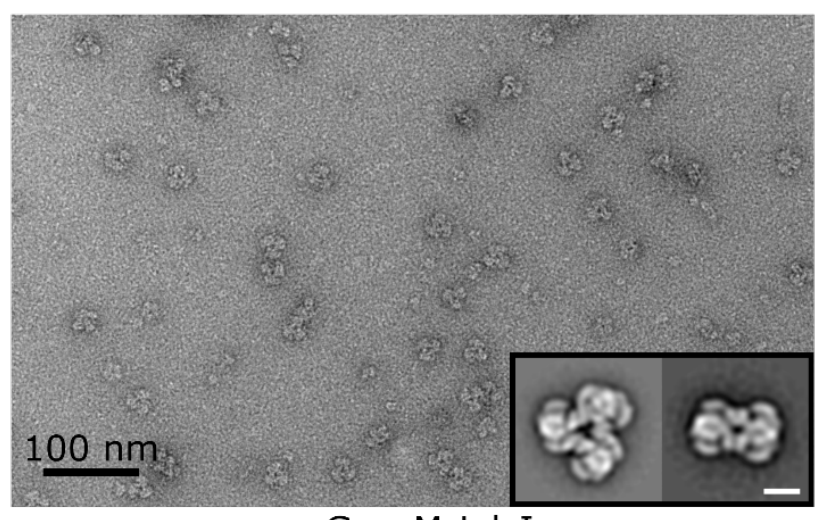

mGeosM-LdcI

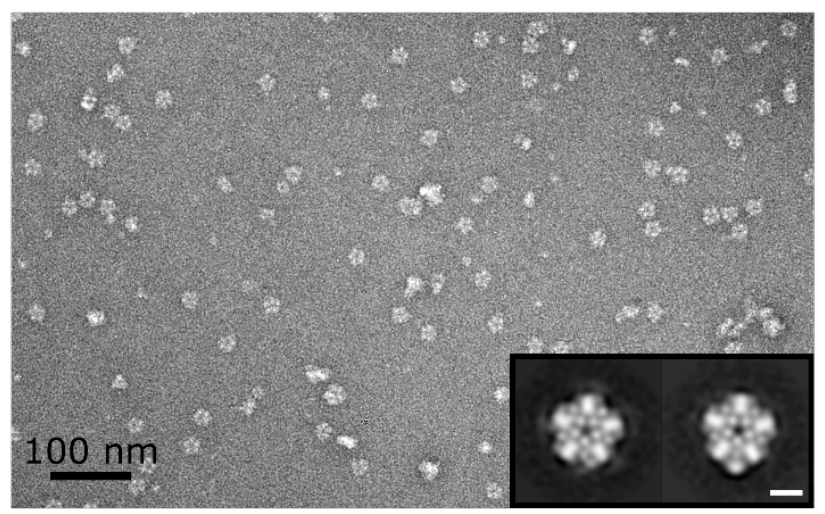

LdcI-mGeosM

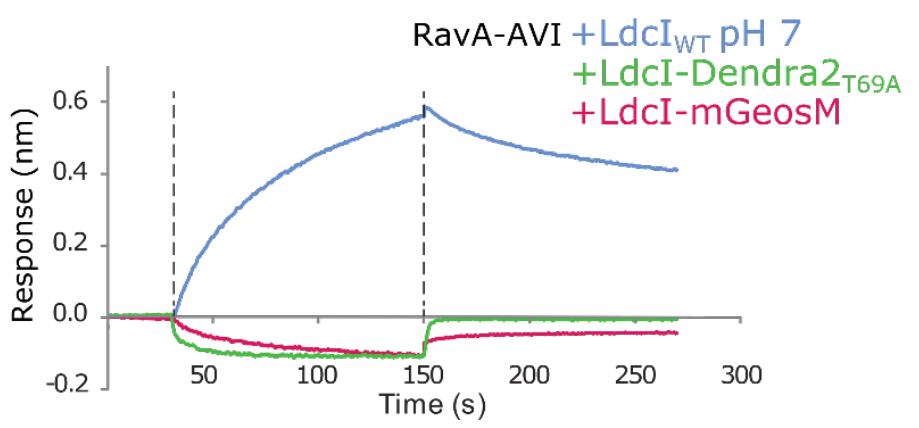

Supplementary Figure 1. Characterisation of Dendra2 ${ }_{\text {T69A-Ldcl and mGeosM-Ldcl fusion }}$ constructs by negative stain EM and BLI. A) Ns-EM micrographs of N-terminal FP-Ldcl fusions Dendra2 ${ }_{\text {r69A }}$-Ldcl (left) and mGeosM-Ldcl (right), with 2D class averages inset (scale bar $=100 \AA$ ). Dendra2 ${ }_{\text {T69A }}-\mathrm{Ldcl}$ only forms dimers, compared to $\mathrm{mGeosM-Ldcl}$ which forms large non-native dodecamers. B) Ns-EM micrographs of C-terminal Ldcl-FP fusions Ldcl-Dendra2 ${ }_{\text {T69A }}$ (left) and Ldcl-mGeosM (right), with 2D class averages inset (scale bar $=100 \AA$ ). Both fusions form decamers, with flexible-linked fluorophores visible as weak densities around the outside of the decameric ring in the 2D class averages. C) BLI binding curves of wild-type $\mathrm{Ldcl}$ (left, [ $\left.\mathrm{Ldcl}_{\mathrm{WT}}\right]=$ 500, 250, 125, 62.5, and $31.25 \mathrm{nM}$ ) and Ldcl ${ }_{W T}$ (right, $500 \mathrm{nM}$ ), Ldcl-Dendra2T69A-Ldcl (right, 500 $\mathrm{nM}$ ) and Ldcl-mGeosM-Ldcl (right, $500 \mathrm{nM}$ ) against RavA-AVI. In contrast to wild-type Ldcl, which binds RavA-AVI with a $46.3 \mathrm{nM}$ affinity, there is no measurable interaction between either of the Cterminal Ldcl-FP fusions and RavA-AVI. 


\section{Supplementary Figure 2.}
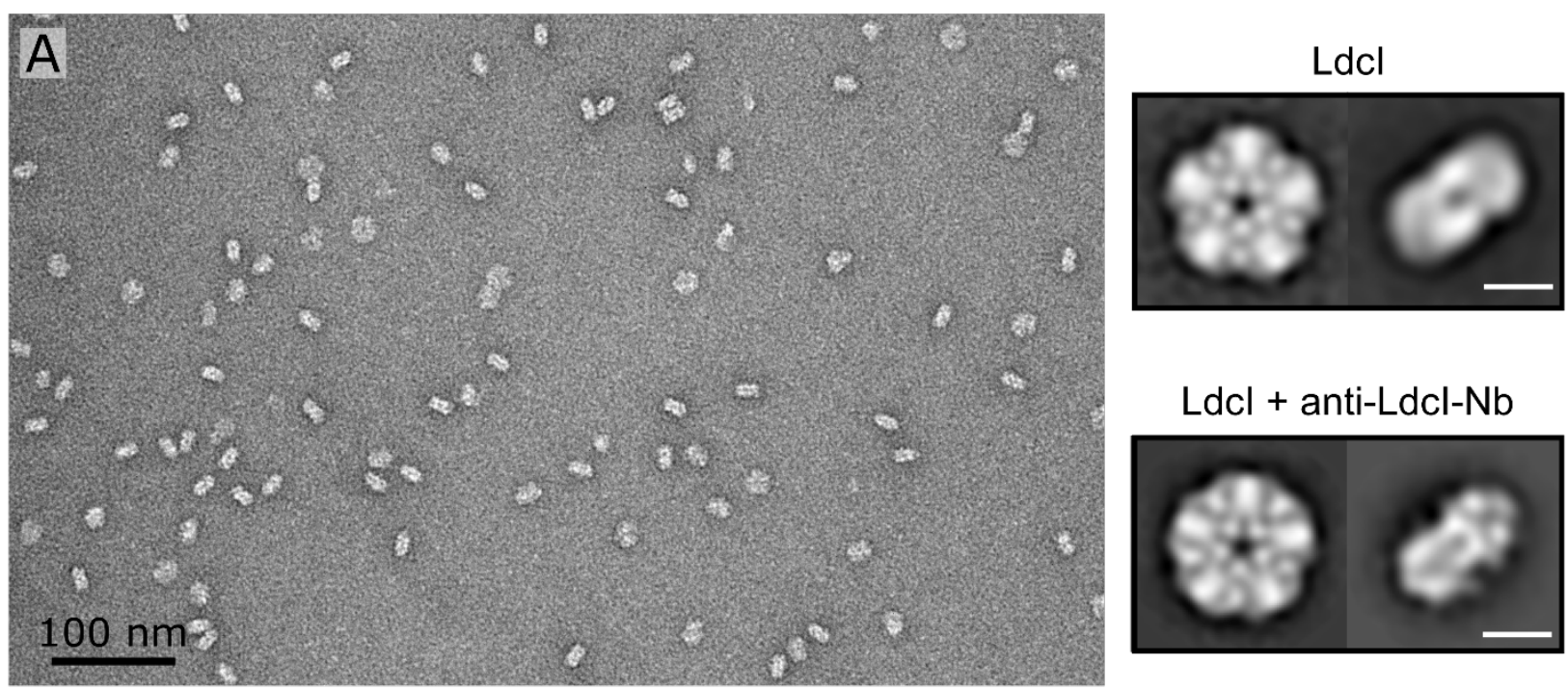

B
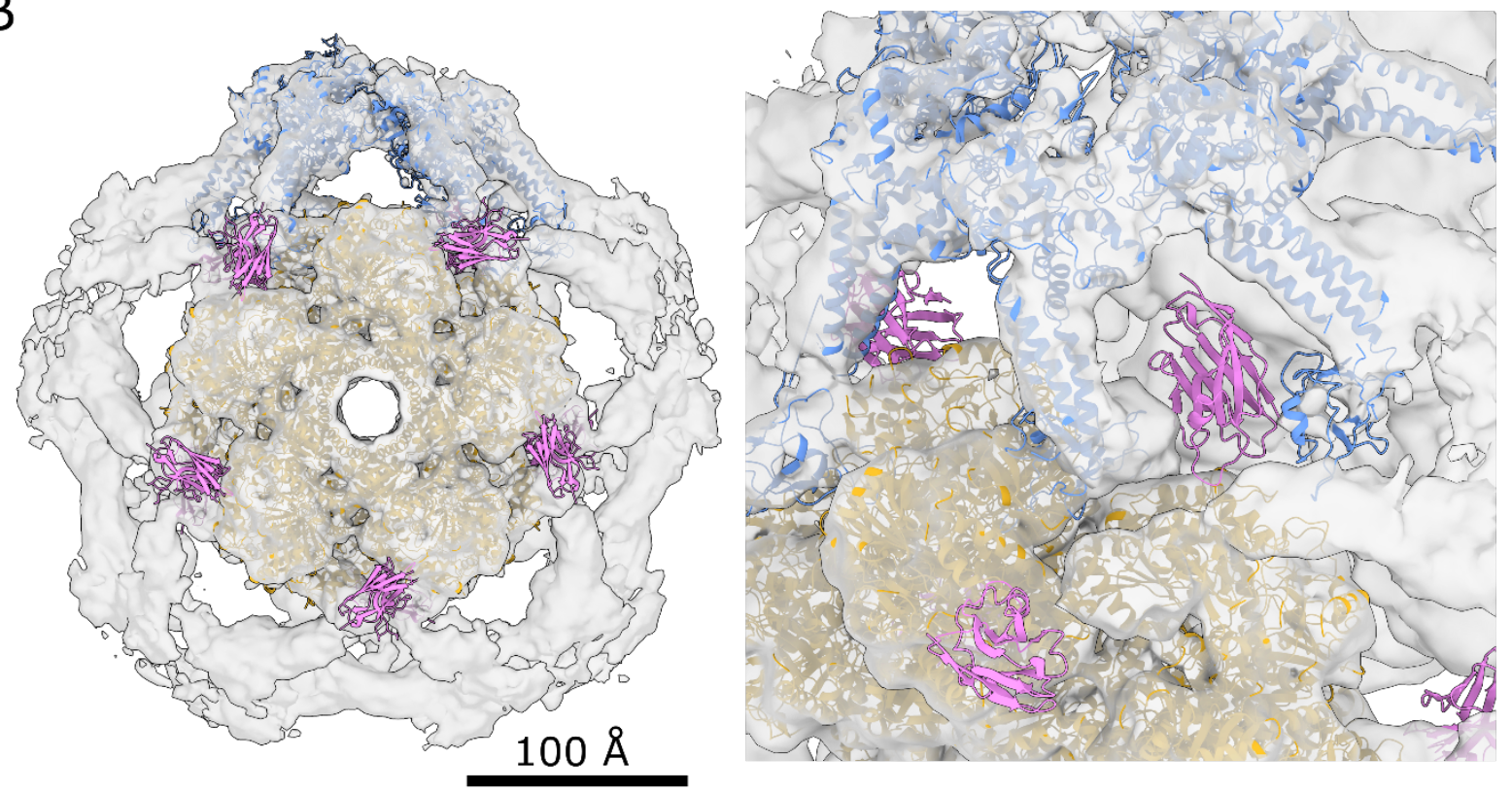

Supplementary Figure 2. Ldcl interacts with anti-Ldcl-Nb and RavA at distinct sites. A) NsEM micrograph of Ldcl + anti-Ldcl-Nb (left), with 2D class averages (right) of Ldcl alone and Ldcl + anti-Ldcl-Nb (scale bar = $100 \AA$ ). B) In silico model of anti-Ldcl-Nb (represented by the antilysozyme nanobody crystal structure, PDB ID: 1MEL) superimposed over the cryo-EM map of the Ldcl-RavA complex (Jessop et al., 2020) (EMDB ID: 4469, PDB ID: 6Q7L). The nanobody (coloured magenta) would occupy a distinct location to the RavA binding site in the Ldcl-RavA cage-like complex (coloured gold and blue). 


\section{Supplementary Figure 3.}
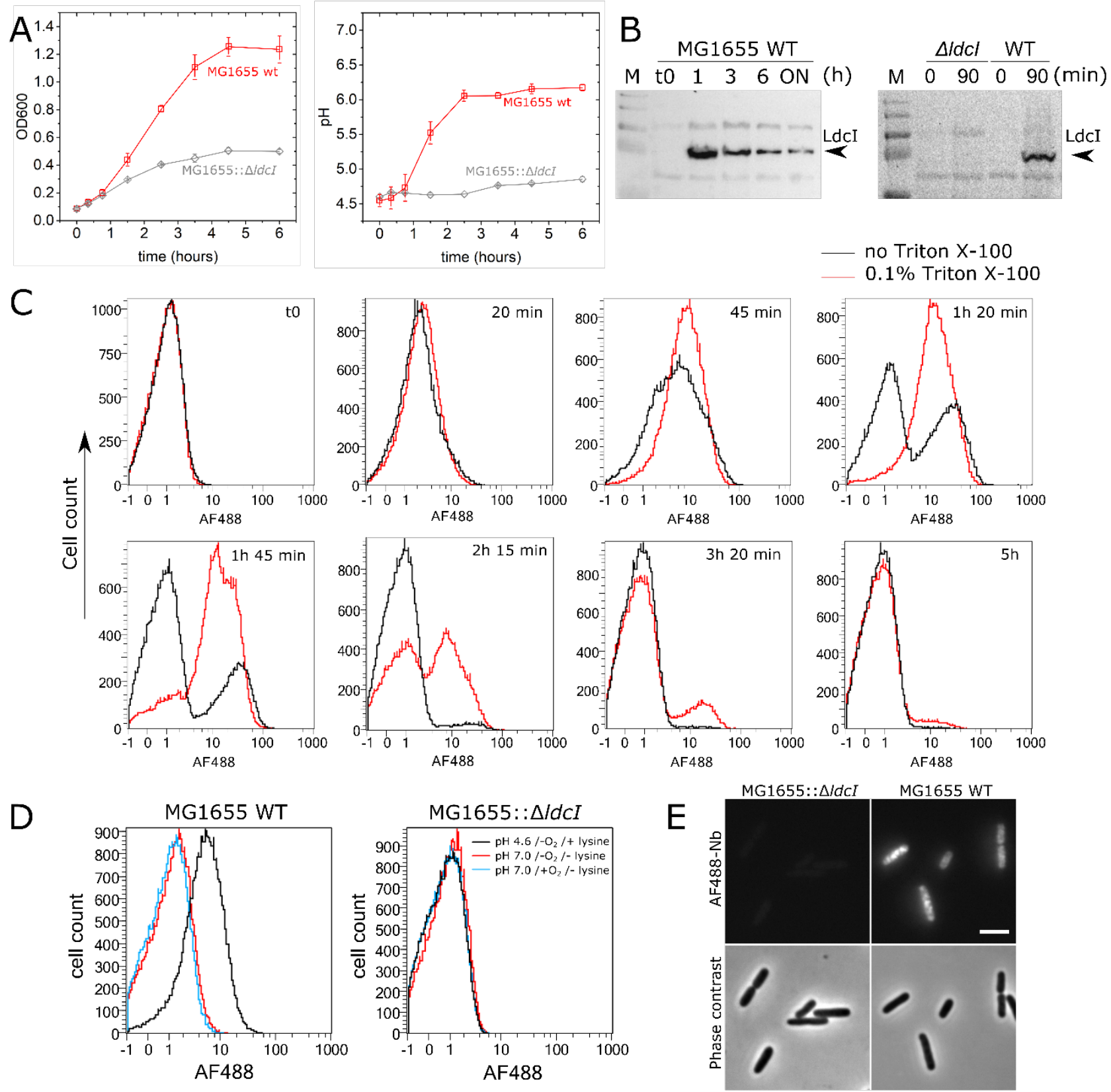

Supplementary Figure 3. Immunolabelling of endogenous Ldcl in E. coli upon acid stress. Characterisation of Ldcl expression using anti-Ldcl-Nb. A) Left - growth curve of wild-type (red) and Ldcl knock-out (grey) cells after exposure to acid stress ( $\mathrm{pH} 4.6$ ), showing compromised growth in Ldcl KO cells. Right - measured $\mathrm{pH}$ of the growth medium for wild-type and Ldcl KO cells after exposure to acid stress ( $\mathrm{pH}$ 4.6). Wild-type cells are able to buffer the medium under acid stress, whereas Ldcl knock-out cells are not. Each data point represents the average of 3 independent measurements taken from 2 biological replicates, error bars correspond to the calculated standard deviation. B) Left - western blot showing Ldcl expression after exposure to acid stress after $0,1,3$ and 6 hours, and overnight (ON). Ldcl expression is maximal soon after exposure to acid stress, tailing off after three hours. Right - western blot against Ldcl in $\Delta / d c l$ and WT MG1655 cells, 0 minutes and 90 minutes after exposure to acid stress, showing a lack of Ldcl expression in $\Delta / d c l$ cells and a lack of baseline expression before exposure to acid stress in WT cells. $\mathrm{M}=$ molecular weight marker. $\mathbf{C}$ ) Flow cytometry measurements carried out after exposure of wild-type cells to acid stress show that Ldcl is maximally expressed one to two hours after exposure to acid stress, before tailing off. D) Flow cytometry measurements of wild-type and Ldcl knock-out cells labelled with anti-Ldcl-Nb-AF488 after 90 minutes of growth under three conditions $-\mathrm{pH} 4.6$ in the absence of oxygen and lysine, neutral $\mathrm{pH}$ in the absence of oxygen and lysine, and 
neutral $\mathrm{pH}$ in the presence of oxygen but absence of lysine. No fluorescence is seen above baseline for the Ldcl knock-out cells under any condition. For wild-type cells, fluorescence is only seen under acid stress conditions. E) Wide-field fluorescence microscopy of Ldcl knock-out MG1655 cells (left) and wild-type MG1655 cells (right), 90 minutes after exposure to acid stress. The staining of anti-Ldcl-Nb-AF488 is specific, shown by the lack of background in the knock-out strain. Scale bar $=2 \mu \mathrm{m}$. 


\section{Supplementary Figure 4.}
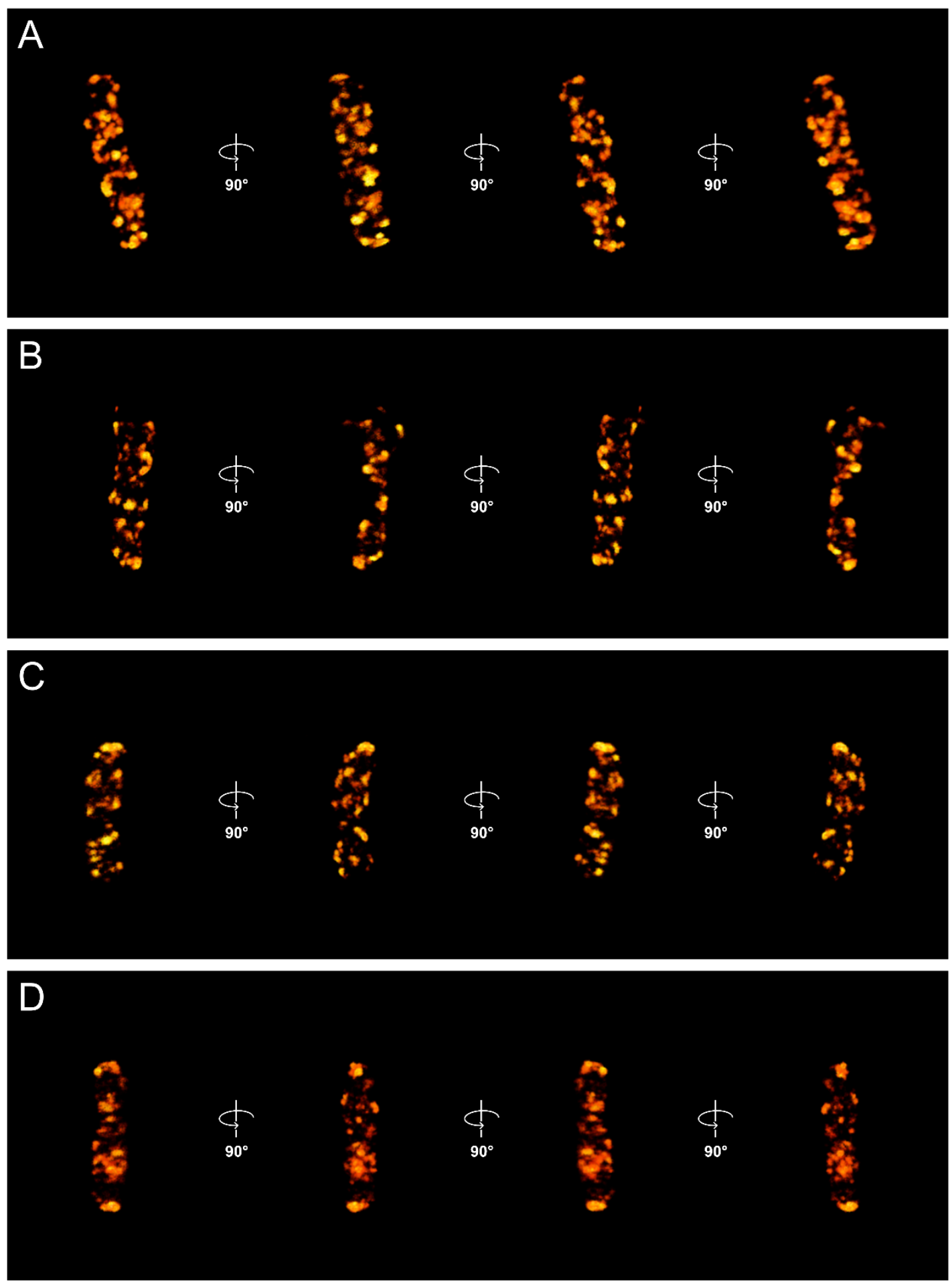

Supplementary Figure 4. Galleries of 3D-STORM images of the four individual E. coli cells shown in Figure 3. Different orientations of each cell related by $90^{\circ}$ rotations around the long axis of the bacterium are shown in order to facilitate visualisation of the patchy distribution of Ldcl. 
bioRxiv preprint doi: https://doi.org/10.1101/2020.05.12.090381; this version posted July 9, 2020. The copyright holder for this preprint (which was not certified by peer review) is the author/funder, who has granted bioRxiv a license to display the preprint in perpetuity. It is made available under aCC-BY-ND 4.0 International license.

\section{Supplementary Figure 5.}

A

Processing flowchart Ldcl decamer ( $\mathrm{pH}$ 7.0)

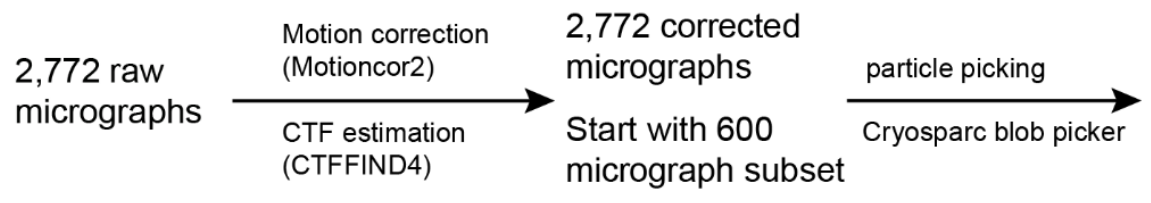

$\sim 796,000$
picked particles
\[ \begin{array}{l}\text { Cryosparc } 2 \mathrm{D} \\ \text { classification }\end{array} \]

Cleaned dataset with (D5) 428,000 particles

template based particle picking on full dataset $3 D$ reconstruction

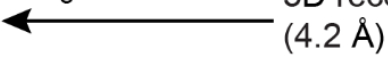

CryoSPARC heterogeneous 3D refinement

(D5)

$3 \mathrm{D}$ reconstruction

$\sim 229,000$ particles

$$
\begin{aligned}
& \text { CryoSPARC } \\
& \text { homo- } \\
& \text { geneous 3D } \\
& \text { refinement (D5) }
\end{aligned}
$$

Ldcl decamer final map, EMD-10849

$2.76 \AA, \sim 229,000$ particles
238,000 picked particles

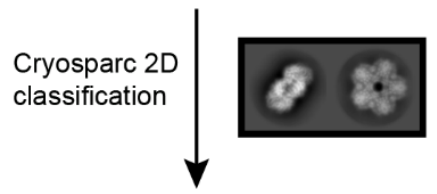

Cryosparc initial

3D refinement (D5) Cleaned dataset with

starting model:

Ldcl decamer (EMD-3204)

filtered to $30 \AA$
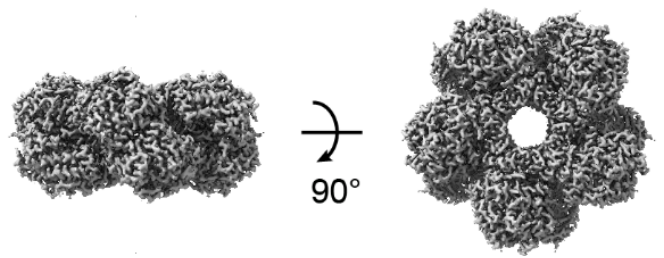

B

Processing flowchart Ldcl stack $(\mathrm{pH} 5.7)$

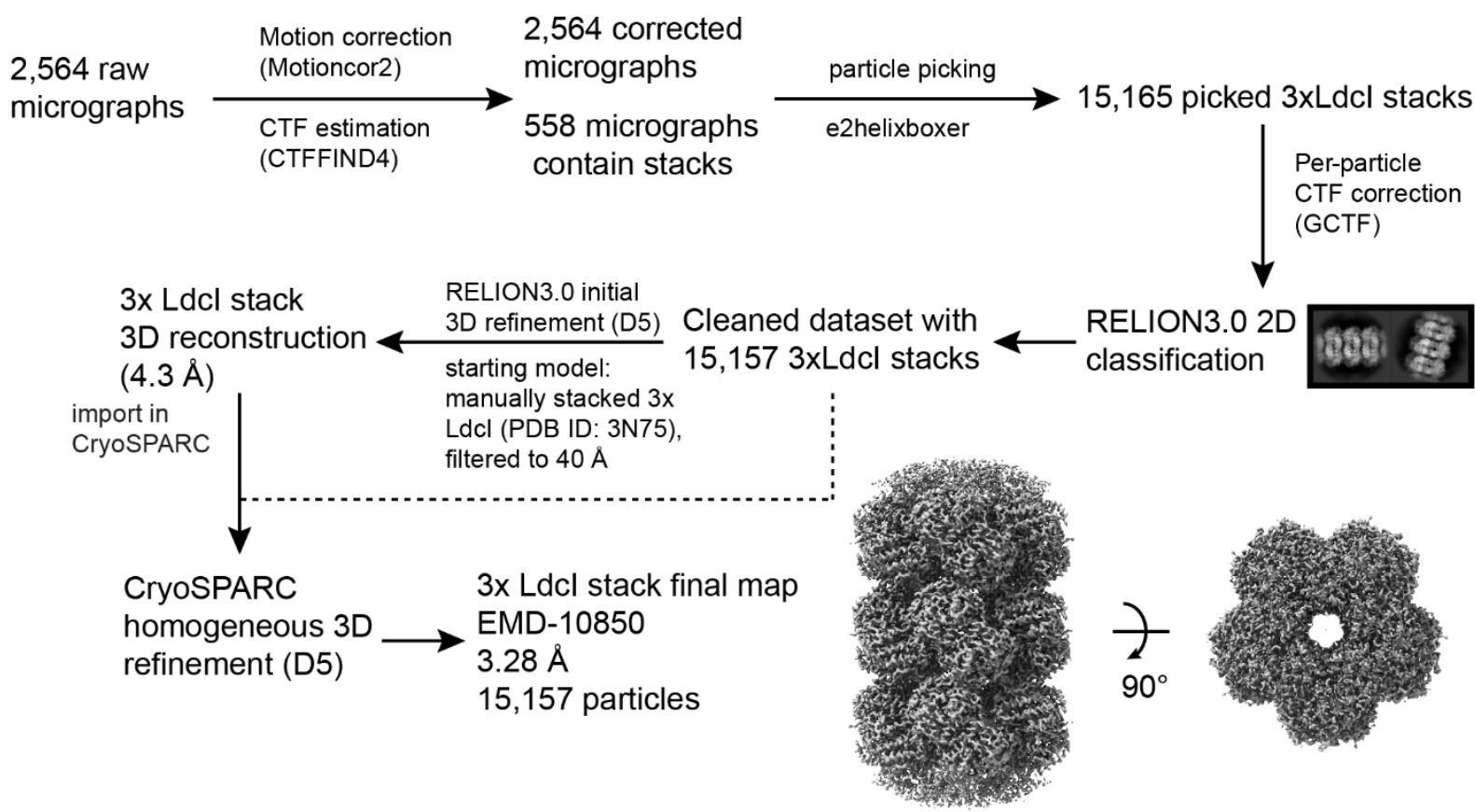

Supplementary Figure 5. Cryo-EM processing pipelines. A) Cryo-EM processing pipeline for Ldcl decamers at $\mathrm{pH}$ 7.0. Unless otherwise indicated, processing steps were carried out in CryoSPARC. B) Cryo-EM processing pipeline for Ldcl stacks at pH 5.7. Software packages used at each step are indicated. For a summary of data collection and processing parameters, and refinement and validation statistics, see Supplementary Table 1. 
bioRxiv preprint doi: https://doi.org/10.1101/2020.05.12.090381; this version posted July 9, 2020. The copyright holder for this preprint (which was not certified by peer review) is the author/funder, who has granted bioRxiv a license to display the preprint in perpetuity. It is made available under aCC-BY-ND 4.0 International license.

\section{Supplementary Figure 6.}

\section{A $\quad$ Ldcl decamer $(\mathrm{pH} 7.0)$}

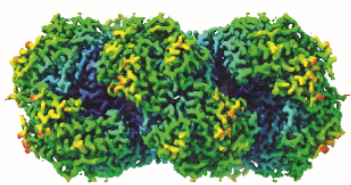

Front

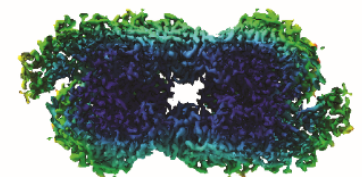

Front (clip)
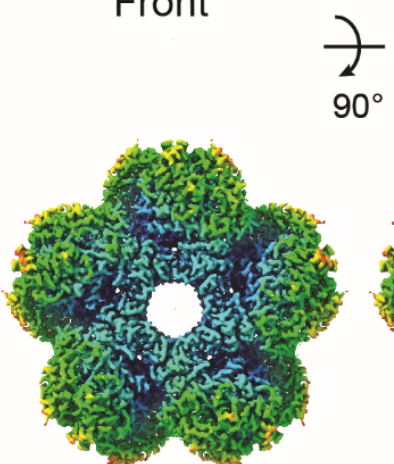

Top

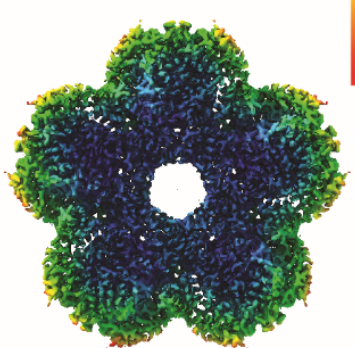

Top (clip)

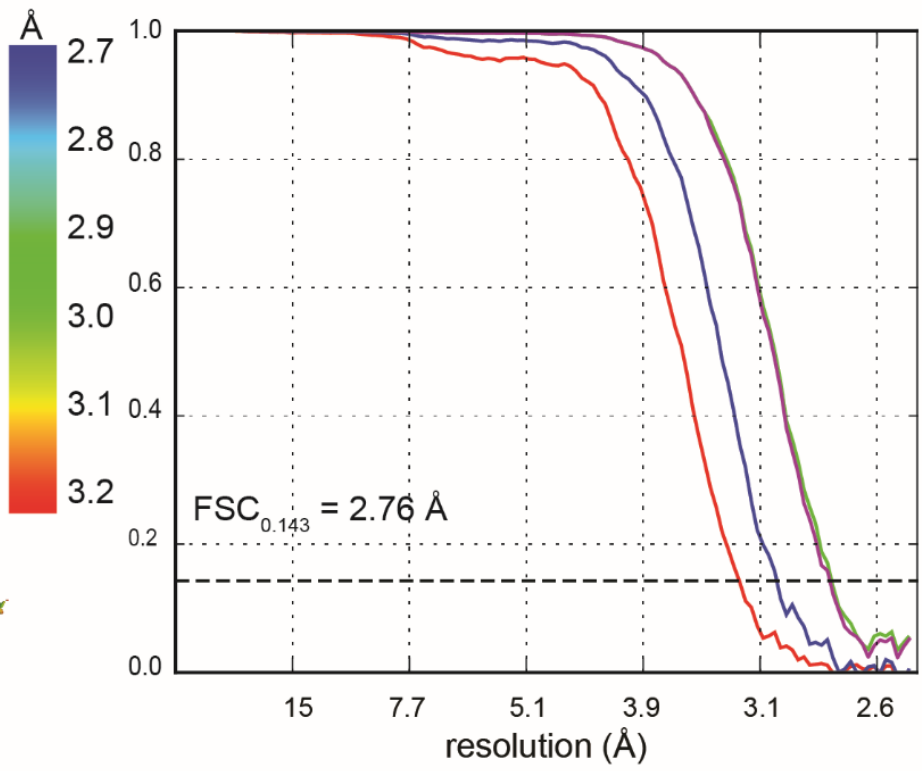

— FSC unmasked

- FSC masked

— FSC spherical mask FSC corrected

\section{B $\quad$ Ldcl stack (pH 5.7)}
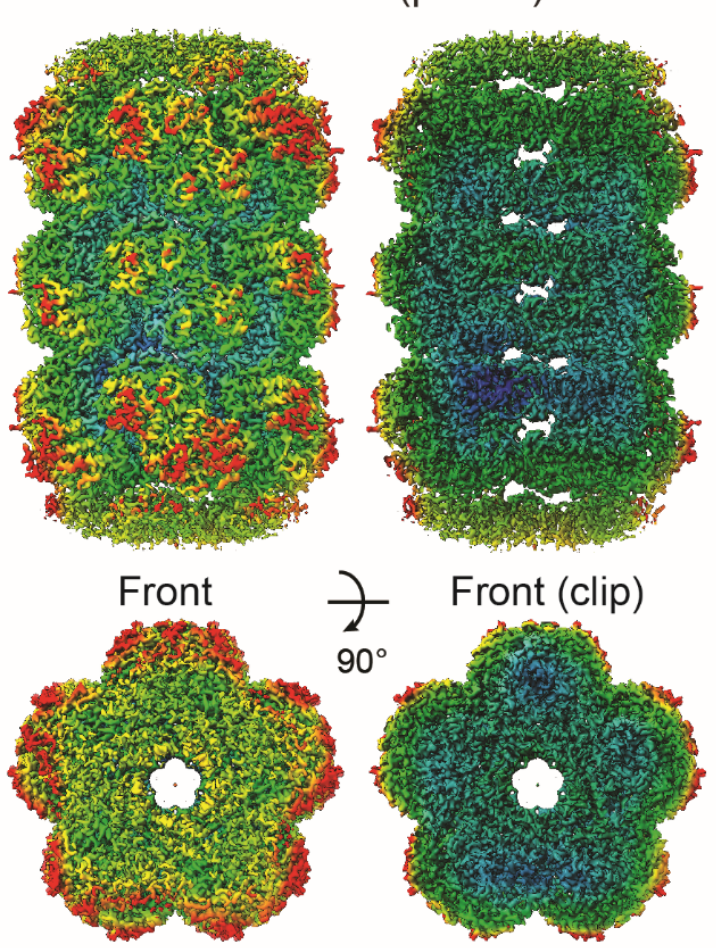

Top
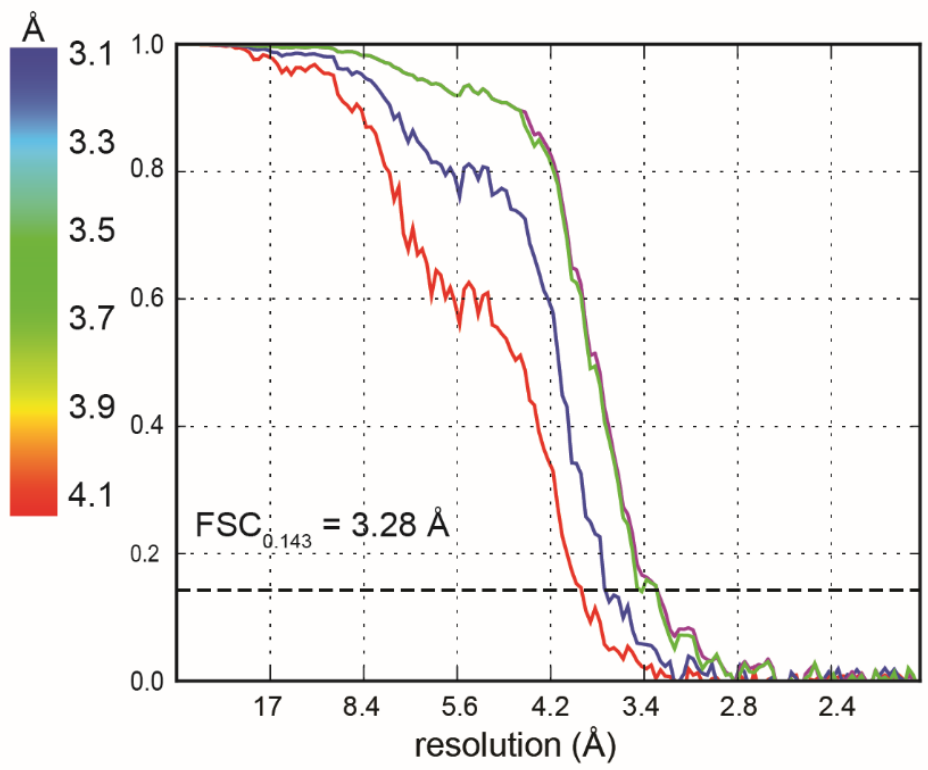

- FSC unmasked

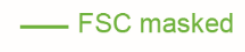

- FSC spherical mask

Supplementary Figure 6. Assessment of the resolution of the cryo-EM maps. 3D reconstructions of $\mathbf{A}$ ) the $\mathrm{Ldcl}$ decamer at $\mathrm{pH} 7.0 \mathrm{~B}$ ) the $\mathrm{Ldcl}$ stack at $\mathrm{pH} 5.7$, coloured according to the local resolution. Gold-standard FSC curves with the estimated resolution at FSC $=0.143$ are shown on the right. 


\section{Supplementary Figure 7.}
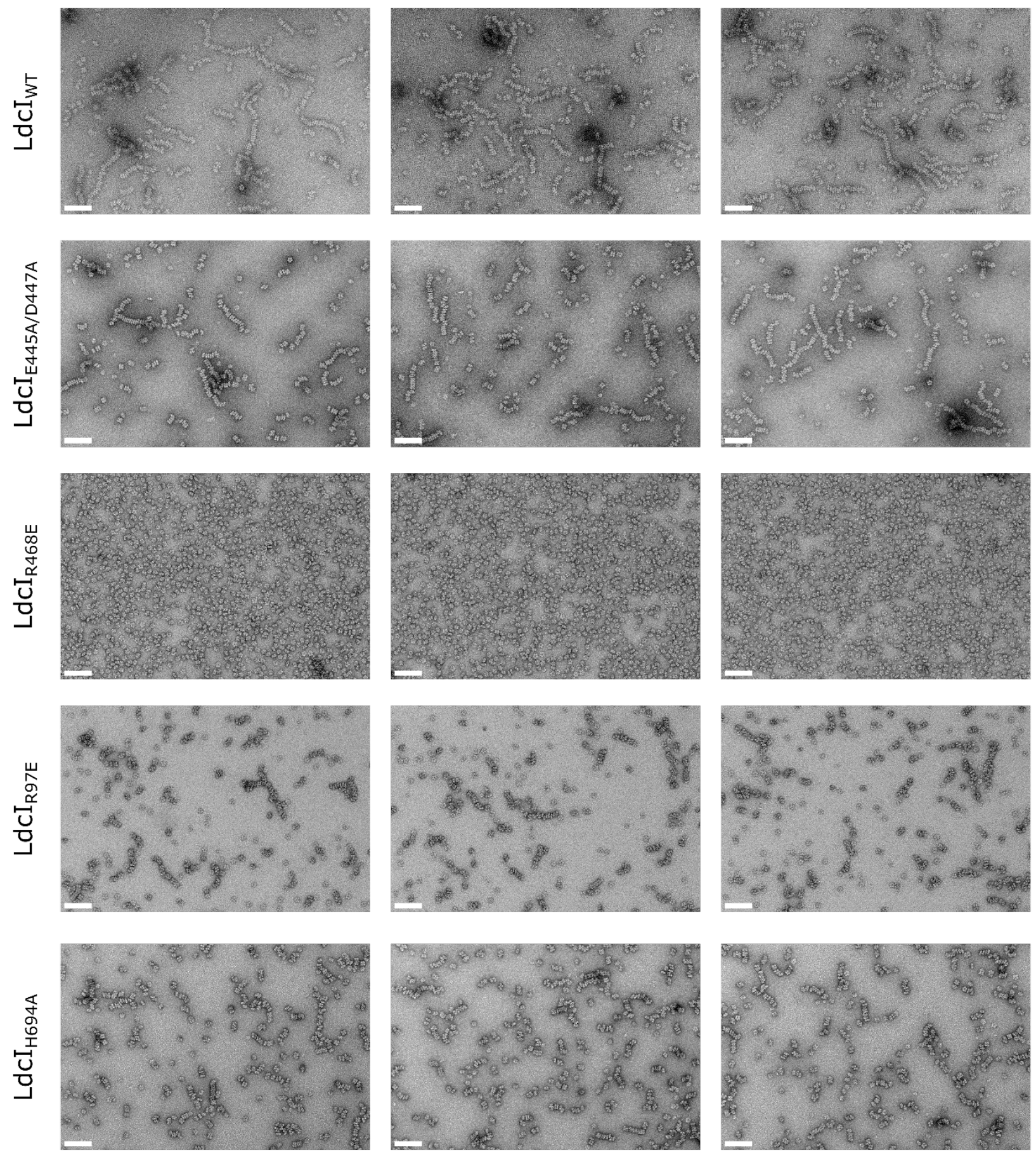

Supplementary Figure 8. Mutational analysis of the predicted molecular determinants of Ldcl polymerisation under acid stress conditions. Full uncropped ns-EM micrographs of wild type and mutant $\mathrm{Ldcl}$ at $\mathrm{pH} 5.7$, scale bars $=100 \mathrm{~nm}$. Three representative micrographs are shown

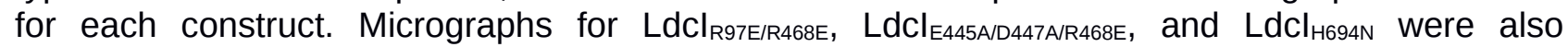
collected but are not shown here. Ldcl E445AID447A/R468E $_{\text {and }}$ Ldcl $_{\text {R97E/R468E }}$ behaved like Ldcl $_{\text {R468E }}$ and remained entirely decameric at low $\mathrm{pH}$, while $\mathrm{Ldcl}_{\mathrm{H} 694 \mathrm{~N}}$ displayed similar behaviour to $\mathrm{Ldcl}_{\mathrm{H} 694 \mathrm{~A}}$. 
Supplementary Table 1. Cryo-EM data collection, refinement and validation statistics

\begin{tabular}{|c|c|c|}
\hline & $\begin{array}{c}\text { Ldcl stacks } \\
(\mathrm{pH} \mathrm{5.7)}\end{array}$ & $\begin{array}{c}\text { Ldcl decamers } \\
(\mathrm{pH} 7.0)\end{array}$ \\
\hline & $\begin{array}{c}\text { EMDB } 10850 \\
\text { PDB 6YN6 }\end{array}$ & $\begin{array}{l}\text { EMDB } 10849 \\
\text { PDB 6YN5 }\end{array}$ \\
\hline \multicolumn{3}{|l|}{ Data collection and processing } \\
\hline Magnification & 130,000 & 116.086 \\
\hline Voltage (kV) & 300 & 200 \\
\hline Electron exposure $\left(\mathrm{e}-/ \AA^{2}\right)$ & 29.3 & 45.0 \\
\hline Defocus range $(\mu \mathrm{m})$ & $1.0-2.5$ & $1.0-3.0$ \\
\hline Pixel size $(\AA)$ & 1.052 & 1.206 \\
\hline Symmetry imposed & D5 & D5 \\
\hline Initial particle images (no.) & 15,165 & $\sim 796,000$ \\
\hline Final particle images (no.) & 15,157 & $\sim 229,000$ \\
\hline Map resolution $(\AA)$ & 3.3 & 2.8 \\
\hline FSC threshold & 0.143 & 0.143 \\
\hline Map resolution range $(\AA)$ & 69.0-3.3 & $51.2-2.8$ \\
\hline \multicolumn{3}{|l|}{ Refinement } \\
\hline Initial model used (PDB code) & $3 N 75$ & $3 N 75$ \\
\hline \multirow{2}{*}{$\begin{array}{l}\text { Model resolution }(\AA) \text { (masked) } \\
\text { FSC threshold }\end{array}$} & 3.7 & 2.9 \\
\hline & 0.5 & 0.5 \\
\hline Model resolution range $(\AA)$ & $\infty-3.7$ & $\infty-2.9$ \\
\hline Map sharpening $B$ factor $\left(\AA^{2}\right)$ & $-96 \AA^{-2}$ & $-173 \AA^{-2}$ \\
\hline \multicolumn{3}{|l|}{ Model composition } \\
\hline Non-hydrogen atoms & 113,760 & 57,074 \\
\hline Protein residues & 14,220 & 7,110 \\
\hline Ligands & 0 & 0 \\
\hline \multicolumn{3}{|l|}{$B$ factors $\left(\AA^{-2}\right)$} \\
\hline Protein & 91.22 & 36.51 \\
\hline Ligand & - & - \\
\hline \multicolumn{3}{|l|}{ R.m.s. deviations } \\
\hline Bond lengths $(\AA)$ & 0.009 & 0.012 \\
\hline Bond angles $\left({ }^{\circ}\right)$ & 1.131 & 1.268 \\
\hline \multicolumn{3}{|l|}{ Validation } \\
\hline MolProbity score & 1.59 & 1.28 \\
\hline Clashscore & 4.78 & 2.83 \\
\hline Poor rotamers (\%) & 0.49 & 0.19 \\
\hline \multicolumn{3}{|l|}{ Ramachandran plot } \\
\hline Favored (\%) & 95.04 & 96.74 \\
\hline Allowed (\%) & 4.96 & 3.26 \\
\hline Outliers (\%) & 0.00 & 0.00 \\
\hline
\end{tabular}




\section{Supplementary Table 2. Root-mean-square-deviation (RMSD) between extracted Ldcl monomer structures at pH 8.5 (PDB ID: 3N75), 7.0 and 5.7.}

\begin{tabular}{l|l|l|l} 
& Ldcl decamer, pH 8.5 & Ldcl decamer, pH 7.0 & Ldcl decamer, pH 5.7 \\
\hline $\begin{array}{l}\text { Ldcl decamer, } \\
\text { pH 8.5 }\end{array}$ & 0 & $0.24 \AA(670$ aligned atoms $)$ & $0.61 \AA$ (610 aligned atoms $)$ \\
\hline $\begin{array}{l}\text { Ldcl decamer, } \\
\text { pH 7.0 }\end{array}$ & $0.24 \AA(670$ aligned atoms $)$ & 0 & $0.56 \AA$ (604 aligned atoms $)$ \\
\hline $\begin{array}{l}\text { Ldcl decamer, } \\
\text { pH 5.7 }\end{array}$ & $0.61 \AA(610$ aligned atoms $)$ & $0.56 \AA(604$ aligned atoms $)$ & 0
\end{tabular}

Supplementary Table 3. Root-mean-square-deviation (RMSD) between extracted Ldcl dimer structures at pH 8.5 (PDB ID: 3N75), 7.0 and 5.7.

\begin{tabular}{l|l|l|l} 
& Ldcl decamer, pH 8.5 & Ldcl decamer, pH 7.0 & Ldcl decamer, pH 5.7 \\
\hline $\begin{array}{l}\text { Ldcl decamer, } \\
\text { pH 8.5 }\end{array}$ & 0 & $\begin{array}{l}0.30 \AA(1328 \text { aligned } \\
\text { atoms })\end{array}$ & $\begin{array}{l}1.51 \AA \text { (1281 aligned } \\
\text { atoms })\end{array}$ \\
\hline $\begin{array}{l}\text { Ldcl decamer, } \\
\text { pH } 7.0\end{array}$ & $\begin{array}{l}0.30 \AA(1328 \text { aligned } \\
\text { atoms })\end{array}$ & 0 & $\begin{array}{l}1.41 \AA(1287 \text { aligned } \\
\text { atoms })\end{array}$ \\
\hline $\begin{array}{l}\text { Ldcl decamer, } \\
\text { pH } 5.7\end{array}$ & $\begin{array}{l}1.51 \AA(1281 \text { aligned } \\
\text { atoms })\end{array}$ & $\begin{array}{l}1.41 \AA(1287 \text { aligned } \\
\text { atoms })\end{array}$ & 0 \\
\end{tabular}


bioRxiv preprint doi: https://doi.org/10.1101/2020.05.12.090381; this version posted July 9, 2020. The copyright holder for this preprint (which was not certified by peer review) is the author/funder, who has granted bioRxiv a license to display the preprint in perpetuity. It is made available under aCC-BY-ND 4.0 International license. 


\section{Supplementary Table 4, A}

\begin{tabular}{|c|c|c|}
\hline Strains or plasmids & Relevant characteristics & Source/reference \\
\hline E. coli Strains & Genotype and Use & \\
\hline BL21(DE3) & $\begin{array}{l}\mathrm{F}^{-} \text {omp T } h s d \mathrm{SB}\left(\mathrm{r}_{\mathrm{B}}^{-}, \mathrm{m}_{\mathrm{B}}^{-}\right) \text {gal } \mathrm{dcm}(\mathrm{DE} 3) \text { : transformation and high level protein } \\
\text { expression using a T7 RNA polymerase-IPTG induction system }\end{array}$ & Novagen product reference 69450 \\
\hline WK6 & $\begin{array}{l}\Delta \text { (lac-proAB) galE strA nal } \mathrm{F}^{\prime}[\text { laclaZ } \triangle \mathrm{M} 15, \text { proAB]: expression of an anti-Ldcl } \\
\text { Nanobody }\end{array}$ & Kindly provided by Dr. Aline Desmyter \\
\hline TOP10 & 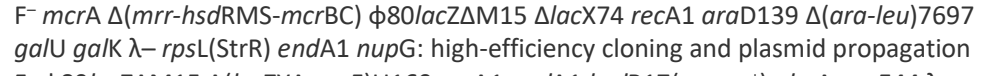 & Invitrogen product reference $\mathrm{C} 404003$ \\
\hline $\mathrm{DH} 5 \alpha$ & $\begin{array}{l}\mathrm{F}^{-} \text {\$80lacZAM15 } \Delta\left(\text { lacZYA-argF)U169 recA1 endA1 hsdR17( }\left(\mathrm{r}^{-}, \mathrm{mk}^{+}\right) \text {phoA supE44 } \lambda^{-}\right. \\
\text {thi-1 gyrA96 relA1: strain used for general cloning and sub-cloning applications }\end{array}$ & Invitrogen product reference 18265017 \\
\hline \multicolumn{3}{|l|}{ Plasmids } \\
\hline \multicolumn{3}{|c|}{$\begin{array}{l}\text { Plasmids for the construction } \\
\text { of Dendra2 }{ }_{T 69 A}-L d c 1 \_p E T / T E V\end{array}$} \\
\hline Ldcl_pET/TEV & $\begin{array}{l}\mathrm{N} \text {-terminal His-tag } \\
\text { ampicillin resistant, high-level prokaryotic expression controlled by the strong } \\
\text { bacteriophage } \mathrm{T7} \text { promoter, produces } \mathrm{T} 7 \text { lysozyme, contains Dendra2 } \mathrm{T} 69 \mathrm{~A} \text { with an } \mathrm{N} \text { - }\end{array}$ & This work \\
\hline Dendra2t69A_pRSETB & terminal His-tag & J Am Chem Soc. 2016 Jan 20;138(2):558-65. doi: 10.1021/jacs.5b09923. Epub 2016 Jan 6. \\
\hline $\begin{array}{l}\text { Result: Dendra2 } \text { T69A- }^{-} \\
\text {Ldcl_pET/TEV }\end{array}$ & $\begin{array}{l}\text { kanamycin resistant, bacterial expression vector, contains a Dendra2 } 269 \mathrm{~A}-\mathrm{Ldcl} \text { fusion } \\
\text { with an } \mathrm{N} \text {-terminal His-tag and a TEV cleavage site between Dendra2 } 699 \mathrm{~A} \text { and Ldcl }\end{array}$ & 然 \\
\hline \multicolumn{3}{|c|}{$\begin{array}{l}\text { Plasmids for the construction } \\
\text { of } m G e o s M\end{array}$} \\
\hline & $\begin{array}{l}\text { ampicillin resistant, high-level prokaryotic expression controlled by the strong } \\
\text { bacteriophage } T 7 \text { promoter, produces } T 7 \text { lysozyme, contains mEos } 2 \text { with an } \mathrm{N} \text { - }\end{array}$ & \\
\hline mEos2-pRSETA & terminal His-tag & $\begin{array}{l}\text { Nat Methods. } 2009 \text { Feb . 6(2):131-3. doi: } 10.1038 / \text { nmeth.1296 } \\
\text { A bright and photostable photoconvertible fluorescent protein. McKinney SA, Murphy CS, } \\
\text { Hazelwood KL, Davidson MW, Looger LL. Nat Methods. } 2009 \text { Feb . 6(2):131-3. } \\
\text { 10.1038/nmeth.1296 }\end{array}$ \\
\hline \multicolumn{3}{|c|}{$\begin{array}{l}\text { Plasmids for the construction } \\
\text { of } m G e o s M-L d c I \text { PET/TEV }\end{array}$} \\
\hline mGeosM-pRSETA & $\begin{array}{l}\text { ampicillin resistant, high-level prokaryotic expression controlled by the strong } \\
\text { bacteriophage T7 promoter, produces T7 lysozyme, contains mGeosM with an } \mathrm{N} \text { - } \\
\text { terminal His-tag } \\
\text { kanamycin resistant, bacterial expression vector, contains Ldcl with a TEV cleavable }\end{array}$ & $\begin{array}{l}\text { Proc Natl Acad Sci U S A. } 2012 \text { Mar 20;109(12):4455-60. doi: 10.1073/pnas.1113770109. } \\
\text { Epub } 2012 \text { Feb } 28\end{array}$ \\
\hline $\begin{array}{l}\text { Ldcl_pET/TEV } \\
\text { Result: } \text { mGeosM- }\end{array}$ & $\begin{array}{l}\text { N-terminal His-tag } \\
\text { kanamycin resistant, bacterial expression vector, contains an mGeosM-Ldcl fusion }\end{array}$ & This work \\
\hline Ldcl_pET/TEV & with an $\mathrm{N}$-terminal His-tag and a TEV cleavage site between mGeosM and Ldcl & This work \\
\hline
\end{tabular}


Plasmids for the construction

of $L d c l-$

Dendra2 T69A_pET22b(+)

Ldcl-Dendra2T69A_pET/TEV

Dendra2T69^_PRSETB

Ldcl_pET22b ++

Result: Ldcl-

Dendra2T69A_pET22b(+)

Plasmids for the construction

of Ldcl-mGeosM_pET22b(+)

Ldcl-Dendra2T69A_pET/TEV

mGeosM-Ldcl_pET/TEV

see above

see above

Ldcl_pET22b(+)

Result: $L d c l-$

mGeosM_pET22b(+)

see above kanamycin resistant bacterial expression vector, contains an Ldcl-Dendra2 2 Tge fusion with a TEV cleavable N-terminal His-tag

\section{This work}

J Am Chem Soc. 2016 Jan 20;138(2):558-65. doi: 10.1021/jacs.5b09923. Epub 2016 Jan 6

kanamycin resistant, bacterial expression vector, contains an Ldcl-Dendra2T69A fusion with a non-cleavable C-terminal His-tag

EMBO J. 2011 Mar 2;30(5):931-44. doi: 10.1038/emboj.2011.5. Epub 2011 Jan 28

This work

This work

This work

kanamycin resistant, bacterial expression vector, contains an Ldcl-mGeosM fusion with a non-cleavable C-terminal His-tag
EMBO J. 2011 Mar 2·30(5):931-44. doi: 10.1038/emboj.2011.5. Epub 2011 Jan 28

This work 


\section{Supplementary Table 4, B}

\begin{tabular}{|c|c|}
\hline Primers & Sequences $\left(5^{\prime}-3^{\prime}\right)$ \\
\hline \multicolumn{2}{|l|}{ Dendra2 ${ }_{\text {T69A-}}-\mathrm{Ldcl}$} \\
\hline V_Ldcl_Dendra2_Fw & TTAATTCCCGGGGTGTTCATGTGATGATGATGATGATGGCTGCT \\
\hline V_Ldcl_Dendra2_Rv & CCCTGCCCAGCCAGGTGTGGAGCAGCGGCGAAAACC \\
\hline I_Dendra2_Ldcl_Fw & GCCATCATCATCATCATCACATGAACACCCCGGGAATTAACC \\
\hline I_Dendra2_Ldcl_Rv & TACAGGTTTTCGCCGCTGCTCCACACCTGGCTGGGC \\
\hline \multicolumn{2}{|l|}{$m E o s 2->m G e o s M$} \\
\hline mEos2-H62M_Fw & ATGTACGGCAACAGGGTATTCGCC \\
\hline mEos2-H62M_Rv & GAATGCAGTGGTCAGGATATCAAAGG \\
\hline mEos2-H62M_2_Fw & CCTGACCACTGCATTCATGTACGGCAACAGGGTATTCG \\
\hline mEos2-H62M_2_Rv & CGAATACCCTGTTGCCGTACATGAATGCAGTGGTCAGG \\
\hline \multicolumn{2}{|l|}{ mGeosM-Ldcl } \\
\hline V_Ldcl_mGeos_Fw & TCTGGCTTAATCGCACTCATGTGATGATGATGATGATGGCTGCT \\
\hline V_Ldcl_mGeos_Rv & TGCCTGACAATGCCAGACGAAGCAGCGGCGAAAACC \\
\hline I_mGeos_Ldcl_Fw & GCCATCATCATCATCATCACATGAGTGCGATTAAGCCAGACA \\
\hline I_mGeos_Ldcl_Rv & TACAGGTTTTCGCCGCTGCTTCGTCTGGCATTGTCAGGCAAT \\
\hline \multicolumn{2}{|c|}{ Ldcl-Dendra2 ${ }_{T 69 A} \&$ Ldcl-mGeosM } \\
\hline CL_Primer_1 & AGCCAGGTGTGGTAAGATCCGGCTGCTAACAAAGC \\
\hline CL_Primer_2 & TGAACCACCGCCTGAGCCTTTTTTGCTTTCTTCTTTCAATACCTTAACGG \\
\hline CL_Primer_3 & GGCTCAGGCGGTGGTTCAGATCTGTACGACGATGACGATAAG \\
\hline CL_Primer_4 & TTACCACACCTGGCTGGG \\
\hline CL_Primer_7 & GGTGGTTCGGGTTCAGGTAACACCCCGGGAATTAACCTG \\
\hline CL_Primer_8 & TGAACCCGATGATCCCGACCACACCTGGCTGGGCAG \\
\hline CL_Primer_25 & GCAAAATGAACGAGAACCTGC \\
\hline CL_Primer_26 & acctgaacccgaaccaccATCTGAACCACCGCCTGAG \\
\hline CL_Primer_27 & tcgggatcatcgggttcaAATTCGAGCTCCGTCGACAAG \\
\hline CL_Primer_28 & GCAGGTTCTCGTTCATTTTGC \\
\hline CL_Primer_19 & gtgagcaaaggcgaagaagatATGAAGATCAAACTCCGTATGGAAGGC \\
\hline CL_Primer_20 & tttatacagttcatccatgccTCCAGAATGAGCAACAGCATGC \\
\hline CL_Primer_21 & atcttcttcgcctttgctcacgtgatgatgatgatgatggctgctg \\
\hline CL_Primer_22 & ggcatggatgaactgtataaaagcagcggcGAAAACCTGTATTTTC \\
\hline CL_Primer_29 & GGTGGTTCGGGTTCAGGTgtgagcaaaggcgaagaagatATG \\
\hline CL_Primer_30 & TGAACCCGATGATCCCGAtttatacagttcatccatgccTCC \\
\hline
\end{tabular}




\section{Supplementary Table 4, C}

\section{Construction of Dendra2T69ALdcl_pET/TEV}

1. PCR using template Ldcl_pET/TEV with primers V_Ldcl_Dendra2_Fw and V_Ldcl_Dendra2_Rv to obtain amplification of the Ldcl containing plasmide

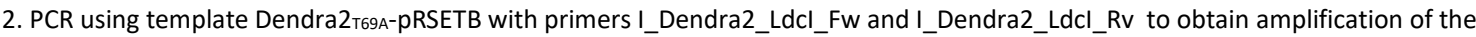
Dendra2т69A gene

3. Gibson assembly using the two obtained fragments

\section{Construction of mGeosM-Ldcl_pET/TEV}

1. Mutagenesis of mEos2 to obtain the mGeosM variant

1. A. PCR on template mEos2-pRSETA using primers mEos2-H62M_Fw and mEos2-H62M_Rv

1. B. Second PCR on the clone obtained in 1.A. with primers mEos2-H62M_2_Fw et mEos2-H62M_2_Rv to obtain mGeosM-pRSETA

2. Construction of an mGeosM-Ldcl fusion

2.A. PCR using template Ldcl_pET/TEV with primers V_Ldcl_mGeos_Fw and V_Ldcl_mGeos_Rv to obtain amplification of the Ldcl containing plasmid

2.B. PCR using template mGeosM-pRSETA with primers I_mGeos_Ldcl_Fw and I_mGeos_Ldcl_Rv to obtain amplification of the mGeosM gene

2.C. Gibson assembly using the two obtained fragments

\section{Construction of Ldcl-Dendra2T69A_pET22b(+)}

1. Construction of Ldcl-Dendra2 ${ }_{\text {T69A_pET/TEV }}$

1. A. PCR using template Ldcl_pET/TEV with primers CL_Primer_1 and CL_Primer_2 to obtain amplification of the Ldcl containing plasmid

1. B. PCR using template Dendra2 $2_{\text {T69 A }}$-PRSETB with primers CL_Primer_3 and CL_Primer_4 to obtain amplification of the Dendra2 ${ }_{\mathrm{T} 69 \mathrm{~A}}$ gene

1. C. Gibson assembly using the two obtained fragments

2. Construction of Ldcl-Dendra2T69A_pET22b(+)

2. A. PCR using template Ldcl-Dendra2 269 __PET/TEV with primers CL_Primer_25 and CL_Primer_26 to obtain amplification of the Ldcl gene

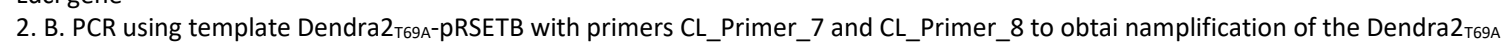
gene

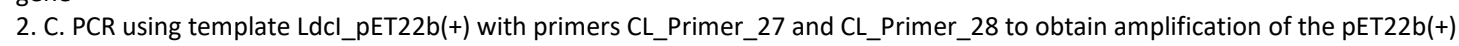
plasmid

2. D. Gibson assembly using the three obtained fragments

\section{Construction of Ldcl-mGeosM_pET22b(+)}

1. Construction of $m$ GeosM-Ldcl_pET/TEV

1. A. PCR using template mGeosM-Ldcl_pET/TEV with primers CL_Primer_21 and CL_Primer_22 to obtain amplification of the Ldcl containing plasmid

1. B. PCR using template mGeosM-pRSETA with primers CL_Primer_19 and CL_Primer_20 to obtain amplification of the mGeosM gene

1. C. Gibson assembly using the two obtained fragments

2. Construction of Ldcl-mGeosM_pET22b(+)

2. A. PCR using template Ldcl-Dendra2T69A_pET/TEV with primers CL_Primer_25 and CL_Primer_26 to obtain amplification of the Ldcl gene

2. B. PCR using template mGeosM-Ldcl _pET/TEV with primers CL_Primer_29 and CL_Primer_30 to obtain amplification of the mGeosM gene

2. C. PCR using template Ldcl_pET22b(+) wih primers CL_Primer_27 and CL_Primer_28 to obtain amplification of the pET22b(+) plasmid

2. D. Gibson assembly using the three obtained fragments 